\title{
Criminologie
}

\section{Délinquance, performance et capital social : une théorie sociologique des carrières criminelles}

\section{Carlo Morselli et Pierre Tremblay}

Volume 37, numéro 2, automne 2004

Délinquance et réussite

URI : https://id.erudit.org/iderudit/010706ar

DOI : https://doi.org/10.7202/010706ar

Aller au sommaire du numéro

\section{Éditeur(s)}

Les Presses de l'Université de Montréal

ISSN

0316-0041 (imprimé)

1492-1367 (numérique)

Découvrir la revue

Citer cet article

Morselli, C. \& Tremblay, P. (2004). Délinquance, performance et capital social : une théorie sociologique des carrières criminelles. Criminologie, 37(2), 89-122. https://doi.org/10.7202/010706ar
Résumé de l'article

Cet article soutient la thèse selon laquelle le capital social des délinquants leur permet d'augmenter de manière significative les revenus qu'ils retirent de leurs activités. La démarche utilisée pour rendre opératoire cette proposition a pour effet de renouveler de différentes façons la sociologie criminelle : il ne suffit pas de savoir si un délinquant « fréquente » d'autres délinquants ou non, il faut mesurer la qualité relationnelle et instrumentale des rapports qu'il entretient avec eux et son aptitude à exploiter les opportunités qu'ils ouvrent ; il ne suffit pas de qualifier les délinquants de « chroniques » ou d'« occasionnels ", il faut plutôt se demander si leur trajectoire délinquante est " réussie » ou non ; et, finalement, il ne suffit pas de décrire les trajectoires délinquantes, il faut en resituer l'analyse dans le contexte plus large des parcours individuels et collectifs de mobilité professionnelle. Un des bénéfices marginaux d'une telle approche est de remettre en cause la thèse selon laquelle les délinquants seraient inaptes au " succès » en raison de leur témérité, de leur impulsivité ou de leur présentisme. Les données de l'étude proviennent d'entrevues auprès d'un échantillon de 156 détenus fédéraux dans le cadre d'une enquête qui s'intéressait à leur situation financière durant les trois années qui avaient précédé leur incarcération actuelle. 


\title{
Délinquance, performance et capital social: une théorie sociologique des carrières criminelles ${ }^{1}$
}

\author{
Carlo Morselli \\ École de criminologie \\ Université de Montréal \\ carlo.morselli@umontreal.ca \\ Pierre Tremblay \\ École de criminologie \\ Université de Montréal \\ pierre.tremblay@umontreal.ca
}

RÉSUMÉ - Cet article soutient la thèse selon laquelle le capital social des délinquants leur permet d'augmenter de manière significative les revenus qu'ils retirent de leurs activités. La démarche utilisée pour rendre opératoire cette proposition a pour effet de renouveler de différentes façons la sociologie criminelle: il ne suffit pas de savoir si un délinquant «fréquente» d'autres délinquants ou non, il faut mesurer la qualité relationnelle et instrumentale des rapports qu'il entretient avec eux et son aptitude à exploiter les opportunités qu'ils ouvrent; il ne suffit pas de qualifier les délinquants de «chroniques» ou d'«occasionnels», il faut plutôt se demander si leur trajectoire délinquante est «réussie» ou non; et, finalement, il ne suffit pas de décrire les trajectoires délinquantes, il faut en resituer l'analyse dans le contexte plus large des parcours individuels et collectifs de mobilité professionnelle. Un des bénéfices marginaux d'une telle approche est de remettre en cause la thèse selon laquelle les délinquants seraient inaptes au «succès» en raison de leur témérité, de leur impulsivité

1. Nous tenons à remercier Julie-Soleil Meeson, Bill McCarthy, Marc Ouimet, Eric Grodsky, Maurice Cusson et Ron Burt pour leurs commentaires à diverses étapes de la rédaction de cet article. L'étude a profité d'une subvention du Conseil de recherches en sciences humaines du Canada et de l'encadrement du Centre international de criminologie comparée de l'Université de Montréal. Les entrevues ont été menées pour l'essentiel par un groupe d'étudiants que nous tenons à remercier : Véronique Parent, Mathieu Charest, Clément Robitaille, Mathilde Turcotte, Katia Petit, Véronique Noël, Cindy Couture, Dave Tanguay, Barbara Wegrzycka, Juliana Hayek et Véronique Lavoie. 
ou de leur présentisme. Les données de l'étude proviennent d'entrevues auprès d'un échantillon de 156 détenus fédéraux dans le cadre d'une enquête qui s'intéressait à leur situation financière durant les trois années qui avaient précédé leur incarcération actuelle.

ABSTRACT - This study follows recent research on criminal earnings and examines the impact of underlying traits (low self-control) and personal organization attributes (non-redundant networking) on criminal earnings amongst a sample of incarcerated offenders previously involved in market and predatory crimes. Controlling for various background factors (age, non-criminal income, lambda, and costs of doing crime), both low self-control and non-redundant networking independently explain why some offenders are more successful than others in achieving higher monetary standards through crime. While efficient, brokerage-like networking enhances market offenders' earnings, low self-control emerges as an asset for predatory offenders: the lower the self-control, the higher their criminal earnings. For market offenders, however, low self-control has no direct effect, but it does mitigate the impact of efficient networking on criminal earnings. The results emerging from this study have implications for Gottfredson and Hirschi's theory of crime and the advent of a criminal network perspective. Extensions are also made towards the conventional/criminal embeddedness framework and deterrence research.

Deux obstacles principaux empêchent de réussir aux affaires: l'hésitation, qui trouble la clarté de l'esprit, et la crainte, qui montre le péril et détourne d'agir. La Folie en débarasse à merveille; mais peu de gens comprennent l'immense avantage qu'il y a à ne jamais hésiter et à tout oser (Érasme, 1987 [1511]: 37).

S'il est vrai que certains délinquants réussissent «mieux» que d'autres dans leurs activités illégales, l'analyse des trajectoires délinquantes gagnerait à s'inspirer des modèles conceptuels en usage dans les études de mobilité sociale. Celles-ci ont pour habitude de définir d'entrée de jeu un critère de "performance», qu'il s'agisse de réussite professionnelle, scolaire ou sociale; puis de mesurer l'inégalité des chances d'atteindre les résultats espérés et de s'interroger ensuite sur les raisons et les conséquences de cet état de choses (voir par exemple Boudon, 1973; Duncan et Hoffman, 1981). Un devis d'analyse assez courant consiste à choisir comme variable dépendante une mesure de réussite professionnelle, à assigner au statut social des parents le rôle de variable antécédente, à introduire le niveau d'instruction des acteurs sociaux comme variable intervenante et à enrichir la discussion en incorporant à titre de variables contextuelles les contraintes de la situation ou de la conjoncture dans laquelle les individus ou les groupes observés se retrouvent (par exemple, l'offre et la demande de qualifications). Il va de soi qu'on peut 
élargir l'éventail des questions et des problèmes susceptibles d'être traités dans un tel schéma en définissant autrement chacune des variables du modèle ou en admettant qu'elles s'influencent mutuellement.

On a souvent essayé de transposer ce paradigme à la sociologie criminelle pour donner une cohérence globale aux recherches sur la criminalité (voir par exemple Cullen et Link, 1980). Mais il est impossible d'y parvenir de manière opératoire sans que certaines conditions préalables soient satisfaites. Puisque la délinquance procure à ceux qui s'y engagent une variété d'avantages, le premier aménagement est d'en mesurer les résultats. Une exigence apparemment banale mais qui présuppose que les individus, délinquants compris, agissent globalement de manière stratégique (Cusson, 1981; 1986). Au regard de l'inégalité patente des chances sociales, il est peu vraisemblable de supposer que les chances criminelles se distribuent au hasard, ou qu'elles se répartissent de manière égalitaire, ou encore qu'il suffise de tenir compte des délits pour lesquels les sujets ont été arrêtés ou condamnés pour en contrôler statistiquement les effets. C'est ce postulat, qui est adopté implicitement dans la plupart des études de récidive, que nous remettons en cause dans cet article qui s'intéresse spécifiquement au degré de réussite économique individuelle des délinquants, c'est-à-dire aux revenus qu'ils retirent de leurs activités illicites. Il est vrai, comme le remarquent McCarthy et Hagan (2001), que d'autres critères de performance auraient pu être envisagés: les marques spécifiques de reconnaissance, formelles (promotions) ou informelles (félicitations), que suscitent leurs délits dans leur entourage immédiat; la satisfaction personnelle ou le sentiment de compétence qu'ils éprouvent à maîtriser les activités illégales qu'ils pratiquent ou à innover; la capacité de contourner, de neutraliser ou de minimiser les risques inhérents aux entreprises illicites (délation, surveillance, arrestation, emprisonnement). En analysant les revenus illicites individuels d'un échantillon de délinquants, on renonce à embrasser l'ensemble des bénéfices que les individus espèrent s'approprier par la délinquance (pouvoir, richesse, prestige). Il reste que la réussite économique est une préoccupation dominante pour la plupart des gens; qu'elle est plus facile à mesurer que d'autres formes d'avantages ; et qu'en analysant une forme de réussite délinquante (la réussite «économique»), on ouvre aussi la voie à l'étude d'autres formes analogues de réussite.

Le deuxième aménagement qu'il convient de mettre en place pour opérer cette transposition de paradigme est d'admettre que les modéli- 
sations de réussite conventionnelle que l'on trouve dans la sociologie de la mobilité sociale ne peuvent pas être utilisées telles qu'elles pour rendre compte des réussites et des échecs qui ponctuent les trajectoires délinquantes. On ne doit pas s'attendre, par exemple, à ce que les performances scolaires influencent fortement (négativement ou positivement) les chances d'accès aux positions qu'offrent les marchés illicites. Cela est d'autant plus improbable que même dans le marché du travail conventionnel la mobilité professionnelle et la mobilité économique des acteurs ne sont que très imparfaitement congruentes. Comme Jencks (1979) l'a bien souligné, autant il existe une relation forte entre le niveau d'instruction et le statut professionnel des emplois occupés, autant la corrélation entre le degré de scolarité et les revenus des individus reste modeste. La réussite financière individuelle des acteurs sociaux dépendrait au moins autant, selon Jencks, des contacts à leur disposition et de compétences qui n'ont pas grand-chose à voir avec leur milieu familial, leurs habiletés cognitives ou leur performance scolaire, notamment leur entrepreneurship, ou de ce que certains attachent plus d'importance que d'autres à l'argent et sont disposés à renoncer à d'autres formes de satisfactions tangibles ou symboliques pour y parvenir. C'est probablement ce qu'ont toujours compris nombre de délinquants qui, n'éprouvant aucun désir de "considération sociale», au sens habituel du terme, ou aucun intérêt particulier pour la chose académique et le «savoir» des autres, sont néanmoins résolus à essayer de s'enrichir et de réussir dans la vie.

Dans cet article, nous nous inspirons de deux études. La première, celle de Matsueda et al. (1992), fait état des revenus licites et illicites d'un échantillon de délinquants adultes et de leur évaluation du prestige social des emplois «licites» et «illicites» qu'offre leur environnement. La thèse qu'ils vérifient stipule que les délinquants qui accordent davantage de prestige aux emplois illicites (prêteurs, «dealers», planteurs, receleurs, etc.) seront davantage enclins à se laisser tenter par la délinquance, qu'ils y seront plus performants (leurs revenus illicites seront plus élevés) et qu'ils seront, par voie de conséquence, moins enclins à participer au marché du travail ou à consacrer des efforts à la recherche de «bons emplois». Cette proposition semble d'autant plus robuste qu'on peut la vérifier quels que soient l'âge et le sexe des sujets, leurs antécédents de délinquance et d'arrestation, leur niveau d'instruction, ou leur degré de toxicomanie - un ensemble de facteurs dont on sait par ailleurs qu'ils modulent à la hausse la motivation des sujets à s'engager 
dans la délinquance et à la «rentabiliser». En revanche, et même si la grande majorité des délinquants interrogés s'accordent pour tenir en plus haute estime les emplois conventionnels que les emplois que leur offre le milieu criminel, la considération qu'ils peuvent éprouver à titre personnel pour ceux qui occupent ces positions ne semble jouer aucun rôle particulier dans leur décision de s'intégrer ou non au marché du travail.

La deuxième étude pertinente provient de l'enquête très fouillée que Hagan et McCarthy (1997) ont réalisée auprès d'un échantillon de jeunes «squatters» de Toronto. Ils se sont intéressés notamment à leurs revenus licites et illicites (McCarthy et Hagan, 2001) et montrent que les délinquants les plus «débrouillards» présentent un profil commun: ils désirent s'enrichir (sur ce thème, voir aussi Agnew, 1994); leur délinquance ne s'éparpille pas dans toutes les directions; ils manifestent une «compétence naturelle», une notion qu'ils empruntent à Clausen (1991) et qui combine trois traits de caractère principaux : confiance en soi, vivacité d'esprit et fiabilité; finalement, ils sont portés à rechercher la collaboration d'autres délinquants et à l'obtenir en retour - une prédisposition dont on a pu montrer qu'elle contribue aux réussites dans toutes les professions (Argyle, 1991). Ces facteurs sont opérants quels que soient l'âge des jeunes gens, leur degré de scolarité ou le montant des revenus qu'ils retiraient de leurs emploi sur le marché du travail.

Nous retenons de ces travaux l'idée que l'étude des trajectoires délinquantes gagnerait à s'attarder, au-delà de leur cursus conventionnel (niveau d'instruction, statut social des parents, accès aux emplois conventionnels), à la manière dont le cursus de leur délinquance peut rendre compte des différences individuelles de performance. Nous retenons en particulier l'hypothèse selon laquelle le capital social des délinquants, soit leur aptitude à rechercher et à obtenir la collaboration d'autres délinquants, est un facteur important de réussite délinquante. Bien que le thème des «pairs délinquants» reçoive un traitement privilégié dans les manuels de criminologie, on ne s'est guère intéressé à examiner de quelle façon les relations entre délinquants pouvaient avoir un impact sur le degré de réussite de leurs activités illicites ${ }^{2}$. Or, il se

2. Les formes diverses de coopération entre délinquants ont été analysées dans les travaux récents de Haynie (2001) et de Warr (2002) et dans l'ensemble des études de John Hagan et Bill McCarthy, notamment (Hagan, 1994; McCarthy,1996; Hagan et McCarthy, 1997; McCarthy et al., 1998). 
trouve que les recherches sur la mobilité professionnelle ont une pertinence pour la sociologie criminelle. Granovetter (1974) a changé notre manière de comprendre la dynamique des processus de mobilité professionnelle et sociale en s'intéressant de près aux relations qu'un échantillon de jeunes adultes entretenaient avec les personnes qui leur avaient permis de se trouver un emploi : contrairement à ce qu'on aurait pu prévoir, les catalyseurs les plus utiles n'avaient été ni leurs amis proches, ni les membres de leurs familles, mais le bassin des «connaissances» avec lesquelles ceux-ci n'avaient entretenu que des rapports occasionnels et plutôt superficiels (pour un bilan des recherches stimulées par cette notion des weak ties, voir Breiger, 1990). Poursuivant dans la même veine, Burt $(1992 ; 2001 ; 2002)$ a su démontrer que les chances de promotion des employés dans les grandes entreprises augmentaient en fonction inverse du degré de redondance de leurs réseaux de relations. La raison en est que les réseaux «tricotés serré» $\mathrm{ou}$ «fortement connexionnels» - une expression que nous empruntons à Lemieux (1999) - accaparent ceux qui en font partie et restreignent la «circulation» des informations utiles, des points de vue ou des occasions à exploiter. Les employés dont le réseau personnel de relations était «lâchement tricoté » ou faiblement cohésif se trouvaient, en revanche, dans une bonne position pour jouer un rôle de pont, d'intermédiaire ou de courtier entre individus ou groupes de provenances diverses, pour récolter plus rapidement au passage un ensemble d'informations stratégiques qu'ils pouvaient choisir ou non de relayer et tirer de cette situation un avantage personnel tangible (qu'il s'agisse d'influence ou de hausses de salaire). Autrement dit, les individus les plus performants en situation de compétition seraient ceux qui disposent d'un «capital social riche en trous structuraux» (Lemieux, 1999). On devrait par conséquent s'attendre à ce que les délinquants les plus performants ne soient pas ceux qui nécessairement en fréquentent «beaucoup» d'autres, mais ceux qui bénéficient de réseaux de contacts riches en capital social ${ }^{3}$.

3. Il est utile de distinguer, comme Lemieux (1999) l'a proposé, la forme d'un réseau de relations de la richesse de son capital social. Il peut arriver que des réseaux fortement soudés comme les réseaux de parenté ou d'affinité (les «amis délinquants», les "familles délinquantes») ou les réseaux fortement hiérarchisés (les «organisations criminelles», ce que Lemieux qualifie d' «appareils») soient moins riches en opportunités individuelles que des réseaux plus lâches d'entraide ou de clientélisme qui se mobilisent périodiquement autour de projets d'action ponctuels au cours desquels les rôles d'instigateur, de facilitateur ou de partenaire peuvent s'interchanger au gré des circonstances ou des tâches particulières à exécuter. Sur l'importance des courtiers et des facilitateurs dans les recherches sur le crime organisé, 
C'est cette proposition que nous souhaitons analyser empiriquement dans cet article.

Il n'est pas sans intérêt de noter que Hagan et McCathy (2001) admettent que les délinquants les plus performants puissent manifester au départ un «talent» naturel qui les prédispose à un tel résultat. Bon nombre de théories criminologiques, notamment celle de Gottfredson et Hirschi (1990), stipulent cependant que les caractéristiques principales des délinquants chroniques seraient leur faible maîtrise d'eux-mêmes (low self-control), leur témérité, leur impulsivité, leur égocentrisme, leur présentisme ou leur «labilité». On devrait s'attendre par conséquent à ce que la grande majorité des biographies délinquantes, tout particulièrement celles des détenus, soient ainsi ponctuées d'insuccès répétés. Il est possible que cette impulsivité ou cette «anomie psychologique» à la fois diminuent leurs chances de réussite dans leurs activités illégales, les privent de capital social ou les empêchent d'en tirer parti - une hypothèse de travail que ne désavoueraient sans doute pas les théoriciens qui se plaisent à concevoir les délinquants comme des individus déraisonnables, faute de mécanismes d'autorégulation, mais néanmoins rationnels dans les décisions ponctuelles qu'ils prennent (Nagin et Paternoster, 1993; Piquero et Tibbetts, 1996). Nous présentons ici les résultats d'une enquête réalisée auprès de détenus canadiens pour vérifier ces hypothèses.

\section{Données et stratégie d'analyse}

Les données proviennent d'entretiens auprès de 284 détenus fédéraux de 5 établissements situés au Québec (le centre de réception, deux pénitenciers à sécurité minimale et deux autres à sécurité moyenne). Pour ces entretiens, nous avons obtenu l'autorisation de consulter la liste des résidents de chaque établissement au moment de l'entrevue. Un numéro a été attribué à chaque nom et les noms furent tirés au hasard. On demandait alors par interphone aux détenus sélectionnés de se rendre, à tour de rôle, dans les locaux des intervieweurs, et c'est alors

voir Adler (1993), Kleemans et Van de Bunt (1999), Klerks (2001), Zaitch (2002); sur l'importance des relations de clientélisme qu'on y trouve, voir Albini (1971), Ianni (1972), Blok (1974), Block et Chambliss (1981), Haller (1991; 1990), Hess (1998); sur la notion d'interchangeabilité des rôles d'instigateur et de partenaire en délinquance juvénile, voir Warr $(1996 ; 2002)$. 
que les objectifs de l'enquête leur étaient présentés et qu'on sollicitait leur coopération. Nous aurions souhaité les rémunérer à titre symbolique pour leur aide (l'entrevue durait environ deux heures), mais les autorités correctionnelles s'y sont objectées. Dans certains cas $(n=23)$, les sujets nous ont été adressés directement, soit par les détenus qui avaient déjà été interviewés, soit par le personnel. Il est également arrivé que leur participation ait été décidée à l'improviste, en croisant par hasard un détenu dans les corridors. L'objectif du sondage était de se renseigner de manière détaillée, au moyen de calendriers mensuels, sur la situation financière des détenus durant les trois années qui avaient précédé leur incarcération actuelle - la même fenêtre temporelle qu'avaient utilisée les deux enquêtes Rand, réalisées dans les années 1970 (Peterson et Chaiker, 1981; Chaiken et Chaiken, 1982). Pour minimiser les efforts de remémoration et pour assurer une certaine homogénéité à cette cohorte de biographies, les détenus qui, au moment de l'entrevue, étaient en prison depuis huit ans ou plus n'ont pas été sélectionnés.

Pour diverses raisons, les analyses qui sont présentées dans cet article se limitent à un échantillon restreint de 156 sujets. Compte tenu des hypothèses de cette analyse, nous avons écarté les sujets qui n'avaient déclaré retirer aucun revenu d'origine criminelle durant la période des 36 mois et qui n'avaient pas été condamnés pour un délit susceptible d'être économiquement profitable $(\mathrm{n}=50)$. Ont également été écartés les sujets qui présentaient une trajectoire délinquante « solitaire» $(n=49)$, parce que nos mesures de capital social supposent que les réseaux de co-délinquance sont composés au minimum de deux autres personnes. Finalement, certains sujets ont été éliminés soit parce qu'ils avaient abandonné l'entrevue en cours de route (19 questionnaires invalides parce que trop incomplets), soit parce qu'ils avaient omis de répondre aux questions qui faisaient partie d'une échelle destinée à mesurer leur inaptitude à l'autorégulation (13 questionnaires valides mais incomplets pour les fins de cette étude). Bien que le tirage au sort nous assure que l'échantillon est représentatif de la population des détenus qui se trouvent dans les prisons fédérales, notre objectif principal ici est plutôt de vérifier l'hypothèse selon laquelle le capital social des délinquants module directement leur performance (revenus illicites) indépendamment de leurs prédispositions personnelles. Au regard de cette hypothèse, le fait d'éliminer les délinquants «solitaires» ne poserait un problème que si les sujets privés de capital social réussissaient leur délinquance «aussi 
bien » que ceux qui en sont pourvus. En réalité, c'est très loin d'être le cas (leurs revenus illicites médians annuels sont inférieurs à 1000 \$). En éliminant les sujets dont les délits ne présentaient aucun intérêt économique, il en résulte que l'échantillon peut être considéré comme représentatif d'une population d'individus dont la performance peut être évaluée en termes financiers et dont la délinquance est suffisamment sérieuse et persistante pour qu'ils puissent se voir condamner à des peines sévères (sentences présomptives de deux ans ou plus).

\section{Mesurer les revenus criminels}

Les revenus médians annuels des délinquants interrogés ont été de $52000 \$$ par année (voir tableau 1). Parce que la distribution de ces revenus est fortement asymétrique (on observe un écart important entre la médiane et la moyenne), les données ont été normalisées par transformation logarithmique - une procédure courante chez les économistes et dans les études qui se sont intéressées aux revenus des délinquants (Matsueda et al., 1992; Tremblay et Morselli, 2000; McCarthy et Hagan, 2001). Cette procédure a également l'avantage de minimiser les biais de vantardise et elle est préférable aux solutions de rechange interventionnistes (éliminer les sujets qui déclarent des revenus « excessifs » ou fixer a priori un plafond aux revenus qu'ils pourraient gagner). La moyenne géométrique des revenus illicites annuels dans cet échantillon a été de 14021 \$ par année (environ 1000 \$ par mois). Mais lorsqu'on élimine ceux qui n'ont déclaré aucun revenu (10,3\% de l'échantillon), la moyenne géométrique est de 47335 \$ par délinquant, une valeur très proche du revenu médian annuel pour l'ensemble des sujets interrogés.

Jusqu'à quel point peut-on se fier aux délinquants interrogés pour donner un compte rendu fiable et valide de leur propre performance? Il n'est pas aisé de répondre à cette question, même si on voit mal à qui d'autre on pourrait se fier. Trois stratégies ont été utilisées pour évaluer la fiabilité et la validité des revenus illicites autodéclarés. Tout d'abord, ce n'est qu'assez tardivement dans l'entretien que les questions relatives aux revenus criminels ont été introduites. Chaque répondant recevait un calendrier de 36 mois qu'il remplissait avec l'intervieweur. La première étape était celle où le sujet se contentait d'indiquer les mois passés en prison, en probation, en maison de transition et en libération 
TABLEA U 1

Concepts, mesures et statistiques descriptives $(n=156)$

\begin{tabular}{|c|c|c|c|c|}
\hline Variable & Description de la variable & Moyenne & Écart type & $\begin{array}{l}\text { Coeff. de } \\
\text { variation }\end{array}$ \\
\hline Revenus criminels & $\begin{array}{l}\text { Somme des revenus } \\
\text { criminels durant les } \\
36 \text { mois de la période } \\
\text { d'analyse (en } \$ \text { ). }\end{array}$ & 617003,93 & 1937803,40 & $314 \%$ \\
\hline Revenus criminels ajustés & $\begin{array}{l}\text { Logarithmes (base } 10 \text { ) } \\
\text { des revenus criminels } \\
\text { (moyenne géométrique } \\
\text { entre parenthèses). }\end{array}$ & $\begin{array}{r}4,6239 \\
(42 \quad 062,98)\end{array}$ & 1,7851 & $39 \%$ \\
\hline Âge du répondant & $\begin{array}{l}\text { Âge au début de la période } \\
\text { fenêtre (en années). }\end{array}$ & 27,77 & 9,09 & $33 \%$ \\
\hline Revenus conventionnels & $\begin{array}{l}\text { Somme des revenus (emploi, } \\
\text { assistance, au noir) durant } \\
\text { la période d'analyse. }\end{array}$ & 23649,94 & 78416,31 & $332 \%$ \\
\hline $\begin{array}{l}\text { Revenus conventionnels } \\
\text { ajustés }\end{array}$ & $\begin{array}{l}\text { Logarithmes (base } 10 \text { ) } \\
\text { des revenus conventionnels } \\
\text { (moyenne géométrique } \\
\text { entre parenthèses). }\end{array}$ & $\begin{array}{r}3,5658 \\
(3679,59)\end{array}$ & 1,3791 & $39 \%$ \\
\hline $\begin{array}{l}\text { Activité délinquante en } \\
\text { matière de délits contre } \\
\text { les biens (incluant vols } \\
\text { qualifiés) }\end{array}$ & $\begin{array}{l}\text { Lambdas individuels: } \\
\text { nombre de délits contre } \\
\text { les biens que les sujets } \\
\text { déclarent avoir commis } \\
\text { durant les } 36 \text { mois de la } \\
\text { période d'analyse. }\end{array}$ & 346,7 & 2063,48 & $596 \%$ \\
\hline $\begin{array}{l}\text { Activité délinquante } \\
\text { ajustée pour la } \\
\text { délinquance prédatrice }\end{array}$ & $\begin{array}{l}\text { Lambdas ajustés: } \\
\text { logarithmes (base 10) } \\
\text { des lambdas individuels } \\
\text { (moyenne géométrique } \\
\text { entre parenthèses). }\end{array}$ & $\begin{array}{r}0,9510 \\
(8,93)\end{array}$ & 1,1306 & $118 \%$ \\
\hline $\begin{array}{l}\text { Activité délinquante en } \\
\text { matière de délinquance } \\
\text { de marché }\end{array}$ & $\begin{array}{l}\text { Lambdas individuels: } \\
\text { nombre de transactions } \\
\text { illicites auxquelles les sujets } \\
\text { déclarent avoir participé } \\
\text { durant les } 36 \text { mois de la } \\
\text { période d'analyse. }\end{array}$ & 7780,78 & 29828,90 & $383 \%$ \\
\hline $\begin{array}{l}\text { Activité délinquante } \\
\text { ajustée pour la } \\
\text { délinquance de marché }\end{array}$ & $\begin{array}{l}\text { Lambdas ajustés: } \\
\text { logarithmes (base 10) } \\
\text { des lambdas individuels } \\
\text { (moyenne géométrique } \\
\text { entre parenthèses). }\end{array}$ & $\begin{array}{r}1,9602 \\
(91,24)\end{array}$ & 1,7406 & $89 \%$ \\
\hline $\begin{array}{l}\text { Proportion de jours en } \\
\text { prison (ou hospitalisé) } \\
\text { durant la période d'analyse }\end{array}$ & $\begin{array}{l}\text { Nombre de jours } \\
\text { d'incarcération ou } \\
\text { d'hospitalisation/1080. }\end{array}$ & 0,19 & 0,25 & $131 \%$ \\
\hline $\begin{array}{l}\text { Coûts actuels de leur } \\
\text { délinquance }\end{array}$ & $\begin{array}{l}\text { Durée de la sentence } \\
\text { qu'ils purgent actuellement } \\
\text { (en mois). }\end{array}$ & 67,44 & 114,4 & $169 \%$ \\
\hline $\begin{array}{l}\text { Capital social des réseaux } \\
\text { de co-délinquants } \\
\text { des sujets }\end{array}$ & $\begin{array}{l}\text { Nombre de contacts non } \\
\text { redondants de leurs réseaux } \\
\text { personnels de co-délinquance. } \\
\text { (Voir texte pour détails } \\
\text { de la mesure.) }\end{array}$ & 3,92 & 2,71 & $69 \%$ \\
\hline Inaptitude à se contrôler & $\begin{array}{l}\text { Échelle de Grasmick et al. } \\
\text { (1993) en } 23 \text { items (plus le } \\
\text { score est élevé, plus le sujet } \\
\text { est impulsif). }\end{array}$ & 78,43 & 16,98 & $22 \%$ \\
\hline
\end{tabular}


conditionnelle. La deuxième étape consistait à repérer les mois où il avait fait l'objet d'une arrestation et les villes où il avait habité durant cette période, les évènements marquants de sa vie durant cette période (hospitalisation, divorce, naissance d'un enfant, mort d'un proche, perte d'emploi). Suivait alors une vague de questions sur l'évolution mensuelle de la situation financière du sujet (son intégration sur le marché du travail et, le cas échéant, ses emplois successifs; ses revenus mensuels, incluant ses revenus d'assistance sociale ou de chômage). Ce n'est qu'après la mise à plat de la trame évènementielle de leur passé récent que les sujets étaient interrogés sur leurs diverses activités délinquantes au cours de chacun des 36 mois et sur leurs revenus illicites. (Sur cette stratégie de contextualisation des histoires de vie, voir Horney et Marshall, 1991; Horney et al., 1995; MacKenzie et Li, 2002; Uggen et Thompson, 2003.)

La deuxième stratégie a été d'incorporer dans le questionnaire deux mesures de revenus criminels - les revenus déclarés et les revenus estimés (sur cette distinction, voir Charest, 2004). Dans le premier cas, on demandait aux délinquants de spécifier les sommes gagnées, toutes activités délinquantes confondues, pour chacun des mois durant lesquels ils avaient été «actifs» : ce sont leurs revenus déclarés. Dans le deuxième cas, on leur demandait d'indiquer la variété de leurs activités illicites durant cette période, les mois où ils avaient été «actifs» dans l'une ou l'autre de ces activités, la fréquence mensuelle de leurs délits par type d'activité ou de leurs transactions par type de marché - leurs «lambdas» - pour chacun des mois où ils avaient été actifs dans cette pratique criminelle et, pour finir, les gains moyens par délit ou par transaction. En multipliant les lambdas individuels ${ }^{4}$ par leurs gains unitaires, on

4. Blumstein et ses collaborateurs (1986) ont introduit cette expression dans le vocabulaire ou le jargon de la recherche criminologique pour désigner la fréquence habituelle avec laquelle les délinquants "passent à l'acte» au cours d'une période donnée. Il s'agit d'un terme qui est emprunté aux statisticiens qui se sont intéressés aux processus de Poisson, c'est-à-dire les processus où la probabilité qu'un évènement quelconque (crime, accident) se produise une deuxième, une troisième fois, etc. est identique à la probabilité qu'il se produise une première fois. Lorsque cette probabilité augmente de manière constante, le processus est qualifié de contagieux. Lorsqu'elle n'est pas uniforme pour l'ensemble des sujets auxquels cet évènement peut arriver, le processus est qualifié d' "hétérogène». Blumstein et al. (1986) considèrent les distributions des probabilités individuelles de récidive (nouveau délit plutôt que nouvelle arrestation ou condamnation) comme intrinsèquement hétérogènes et s'intéressent tout particulièrement aux questions que soulèvent l'identification, la prédiction et la neutralisation des délinquants présentant une prédisposition élevée à la récidive (bigh rate 
obtenait ainsi leurs revenus estimés, c'est-à-dire les revenus que nous estimions qu'ils avaient obtenus de leurs activités. La corrélation entre les deux mesures de revenus est satisfaisante $(r=0,45)$ - analogue en fait à celle que l'on trouve dans d'autres recherches (Charest, 2004; voir aussi Reuter et al., 1990; Robitaille, 2001) qui croisent des mesures distinctes ou complémentaires des revenus des délinquants. Lorsqu'on examine plutôt les logarithmes de leurs revenus déclarés et estimés, la corrélation est encore plus forte $(r=0,93)$. L'analyse détaillée que Charest (2004) consacre à cette question montre que la divergence entre les revenus déclarés et estimés est principalement imputable à la complexité intrinsèque de la tâche cognitive demandée (qui n'était pas simple) et qu'elles ne dépendaient pas du contexte institutionnel dans lequel se déroulaient les entretiens, de la dynamique de l'entretien luimême (le sexe de l'intervieweur par exemple) ou des caractéristiques individuelles des sujets (leur âge, leur degré de scolarité, leurs antécédents pénaux ou même leur degré d'impulsivité).

La troisième stratégie de validation a été de croiser leur performance déclarée (les revenus que les sujets disaient retirer de leurs délits) et leur propre évaluation de cette performance. Ceux qui jugeaient leur performance délinquante comme étant nulle ou médiocre avaient effectivement déclaré des revenus annuels inférieurs à 2000 \$; ceux qui évaluaient leur performance comme moyenne ou satisfaisante déclaraient des revenus annuels intermédiaires de 54000 \$ et ceux qui estimaient avoir «très bien réussi» rapportaient en moyenne des revenus annuels de 87000 \$. À la question «Quel est le revenu légal suffisant qui vous inciterait à vous ranger? », les sujets ont répondu que ce revenu devrait être en moyenne de 49000 \$ par année. Ceux qui estimaient avoir très bien réussi dans leurs activités illégales étaient prêts à se « retirer» s'ils pouvaient disposer d'un revenu légitime de 63000 \$ par année, alors

offenders). Si le tempo individuel n'est pas considéré comme une caractéristique «personnelle» mais situationnelle, il a des implications importantes pour analyser la criminalité dans son ensemble puisque le volume de délits qui se commettent dans une collectivité dépend à la fois du nombre d'individus qui participent à la délinquance (prévalence ou taux de participation à la délinquance) et du nombre de délits que chacun d'entre eux commet durant cette période (leur «degré d'activité» ou leur «lambda»). Il arrive que la criminalité augmente non pas parce qu'il y aurait plus d'individus qui se laissent tenter par la délinquance, mais simplement parce que les délinquants «déjà là » sont devenus plus «actifs» ou le demeurent plus longtemps. Les causes qui expliquent un changement du taux de participation n'expliquent pas nécessairement les fluctuations de leur degré d'activité. Et vice versa. 
que les moins performants se seraient satisfaits d'un revenu annuel de 33000 \$ à 35000 \$. D'autres études suggèrent également que les délinquants évaluent de manière réaliste leur propre performance (voir, par exemple, Levitt et Venkatesh, 2000; Tremblay et Morselli, 2000).

\section{Mesurer le capital social des réseaux de co-délinquance}

Nous inspirant des sondages qui s'intéressent aux réseaux de relations des personnes interrogées, les détenus ont été priés d'identifier sur une feuille à part (détruite à la fin de l'entrevue) les prénoms de leurs partenaires, de leurs fournisseurs, de leurs «mentors» ou de toute autre personne qui faisaient partie de leurs «contacts» utiles dans le déroulement des activités illégales spécifiques dans lesquelles ils étaient impliqués. La notion de co-délinquance est utilisée ici de manière assez large (Tremblay, 1993) et désigne tous ceux qui contribuent au déroulement et au succès d'une activité délinquante (le receleur pour le cambrioleur, le fournisseur pour le revendeur, celui qui vole le numéro de la carte de débit pour celui qui la «passera» dans les guichets automatiques, etc.). Pour ne pas allonger l'entretien, le nombre admissible de co-délinquants a été fixé à 15 . Comme l'indique le tableau 1 , la taille moyenne des réseaux de co-délinquance des sujets de l'enquête était de 6,6 personnes. L'écart type étant de 3,9 contacts, on peut en conclure que ces réseaux de co-délinquance variaient de 3 à 11 personnes pour les deux tiers des détenus interrogés (ou de 0 à 11, si l'on tient compte des 47 délinquants solitaires qui ont été exclus de l'analyse).

Pour mesurer le capital social individuel des sujets, nous avons examiné la matrice des rapports entre les éléments de chaque réseau: d'une part, les relations entre le sujet du réseau («ego») et l'ensemble de ses «contacts»; d'autre part, les relations qui s'établissent entre les « contacts» d'ego. On a également demandé aux délinquants interviewés de nous renseigner sur la force de chacune de leurs relations ou de leurs «associations» (une expression que l'on emprunte à Sutherland). Nous avons suivi ici la stratégie de codification proposée par Burt (1992: 287-288): une valeur de 100 lorsque les liens entre deux membres du réseau étaient «très proches» et de 69 lorsqu'ils étaient "proches». Toutes les relations « occasionnelles» ou «distantes» recevaient respectivement des cotes de 37 et 1 respectivement lorsqu'elles impliquaient «ego» et de 34 et 0 lorsqu'elles ne l'impliquaient pas. L'analyse des 
matrices a été réalisée en utilisant la version 5.0 d'Ucinet, mise au point par Borgatti et al. (1999).

Au regard de nos hypothèses de départ, nous souhaitions évaluer jusqu'à quel point le capital social des délinquants interrogés était « riche en trous structuraux». La taille «efficace» d'un réseau (effective size), son potentiel effectif d'opportunités, peut se calculer en éliminant ses caractéristiques redondantes et en utilisant la formule dérivée des travaux de Burt (1992) et de Borgatti (1997):

(1) $\left[\mathrm{n}-\Sigma\left(\left(\mathrm{n}-\mathrm{n}^{\prime}-1\right) / \mathrm{n}\right)\right]$,

où $\mathrm{n}$ désigne le nombre de relations dyadiques potentielles qui peuvent exister entre les contacts d'ego;

et où n' dénombre celles qui ne se sont pas actualisées ${ }^{5}$.

Pour fins d'illustration, supposons que le réseau $\mathrm{E}$ se compose de trois contacts $(\mathrm{n}=3)$ qu'on appellera A, B et C. Supposons que E soit une clique et que les relations entre les membres du réseau soient toutes très fortes (chaque relation ayant une valeur de 100). Comme toutes les relations potentielles entre $\mathrm{A}, \mathrm{B}$ et $\mathrm{C}$ ont été actualisées, le terme «n'» est égal à 0 . $\mathrm{Il} \mathrm{y}$ a trois chemins qui permettent à $\mathrm{A}$ d'être en rapport avec B: celui qui passe par ego, celui qui passe par $\mathrm{C}$ et celui qui le relie directement à $\mathrm{B}$. Mais le chemin qui passe par ego est un chemin par défaut, en ce sens que s'il n'existait pas, A ne pourrait être considéré comme un contact de ego. Il doit donc être déduit d'office du nombre de chemins potentiels qui relient $\mathrm{A}$ à $\mathrm{B}$ [d'où le terme $\mathrm{n}-1$ dans la formule (1)]. Les deux autres chemins qui permettent de relier $\mathrm{A}$ à $\mathrm{B}$ existent de sorte que «n'» est égal à 0 . Le coefficient de redondance que l'on retrouve exprimé par le terme $(n-n$ - 1$) / n$ désigne la proportion de chemins potentiels qui s'avèrent redondants et cette proportion équivaut dans le cas de la relation entre $\mathrm{A}$ et $\mathrm{B}$ à $(3-0-1) / 3=0,666$. La somme des coefficients de redondance des relations $\mathrm{A}$ et $\mathrm{B}, \mathrm{B}$ et $\mathrm{C}$, et $\mathrm{A}$ et $\mathrm{C}-$ l'expression $\Sigma\left(\left(\mathrm{n}-\mathrm{n}^{\prime}-1\right) / \mathrm{n}\right)$ de la formule $(1)$ - sera donc égale à $2(0,666 \times 3)$. D'où il s'ensuit que le capital social du réseau $\mathrm{E}$ aura, selon la formule (1), une valeur égale à $1(3-2)$. La taille «efficace» réelle du réseau $\mathrm{E}$ se résume à 1 parce qu'un seul des contacts d'ego n'est pas redondant.

5. La variable n' peut être pondérée par le temps ou l'énergie que chaque contact d'ego consacre à d'autres membres du réseau. 
Supposons maintenant que le réseau E' soit en tous points similaires à $\mathrm{E}$ à une exception près: $\mathrm{A}$ entretiendrait des liens très forts avec $\mathrm{B}$ mais n'aurait pas de lien avec C. Dans ce cas de figure, on doit déduire des trois chemins potentiels qui relient $\mathrm{A}$ à $\mathrm{B}$ à la fois, le chemin qui passe par ego et le chemin indirect qui passerait par C: le terme «n'» est donc égal cette fois à 2 ; le coefficient de redondance de la relation entre $\mathrm{A}$ et $\mathrm{B}$ équivaut à $0,333((3-1-1) / 3)$, celui de la relation entre $\mathrm{B}$ et $\mathrm{C}$, à 1 et celui de la relation entre $\mathrm{A}$ et $\mathrm{C}$, à 0 . La somme de ces coefficients est donc de 1,333. D'où l'on déduit que le capital social ou la taille «efficace» de E' est réellement de 1,666 (3 - 1,333).

Considérons le dernier cas de figure, celui du réseau E". Il présente les mêmes caractéristiques que $\mathrm{E}$ et $\mathrm{E}^{\prime}$ en ce sens que ego entretient encore une fois des relations très étroites avec ses trois contacts. En revanche, aucun des contacts d'ego ne se connaissent. Ego émerge ici comme le seul pont ou la seule source de raccordement des éléments qui le composent. Comme il n'existe aucune relation entre A ou B ou $\mathrm{C}$, le coefficient de redondance d'une relation qui n'existe pas est nécessairement égal à 0 . La logique de la démonstration est la même que dans les cas précédents. Il existe en principe trois chemins potentiels qui pourraient relier $\mathrm{A}$ à $\mathrm{B}$. De ces trois chemins, celui qui relierait directement A et B n'existe pas et celui qui passerait par C n'existe pas non plus, puisqu'il n'y a aucun lien entre $A$ et $C$ d'une part et $C$ et $B$ d'autre part. Le seul chemin qui permettrait de relier $A$ à $B$ est celui qui passe par ego, mais on a déjà admis qu'il doit être déduit du compte. Le numérateur du terme $\mathrm{n}-\mathrm{n}$ '/n est donc égal à $0(3-2-1)$. Il s'ensuit que le coefficient de redondance est égal 0 , tout comme la somme des coefficients de redondance. Le réseau E" est ici aussi efficace qu'il peut l'être compte tenu de sa taille. Sa taille efficace est effectivement égale à sa taille réelle $(3-0=3)$, parce que les trois contacts d'ego ne sont pas redondants et qu'aucun d'entre eux ne peut accéder aux autres sans passer par ego.

Plus un délinquant accumule les relations dyadiques à faible coefficient de redondance, plus son réseau de co-délinquance est riche en capital social. Cette mesure de capital social varie inversement avec d'autres mesures relationnelles, comme la densité des réseaux personnels $(r=-0,48)$, mais en fonction directe de la taille du réseau des relations de co-délinquance $(r=0,72)$ des sujets. Autrement dit, plus les réseaux sont denses, moins ils sont riches en capital social; plus ils sont étendus, plus les occasions de capitaliser sur ces relations augmentent. 


\section{Variables contrôlées}

L'objectif de cet article est de démontrer que le capital social des délinquants influence leur réussite dans leurs activités illicites et les niveaux de revenus qu'ils peuvent atteindre. Nous souhaitons démontrer que cette influence est directe, qu'elle est spécifique et qu'elle est opérante quels que soient les prédispositions individuelles des délinquants, leur âge, leurs antécédents ou leurs revenus légitimes.

Nous avons incorporé dans le questionnaire administré lors de nos entretiens avec les sujets une échelle de 23 questions mise au point par Grasmick et al. (1993). La fonction de cette échelle était d'opérationnaliser le complexe de traits de personnalité que Gottfredson et Hirschi (1990) jugent caractéristique des délinquants, en l'occurrence leur faible contrôle de soi. Elle comprend six dimensions: impulsivité, goût du risque, préférence pour les activités physiques, égocentrisme, tempérament colérique et inaptitude aux tâches intellectuellement complexes. La cohérence interne de cette échelle est tout à fait satisfaisante (alpha de Cronbach $=0,84$ ) et comparable à celle que l'on obtient dans d'autres enquêtes générales auprès de populations générales, toxicomanes ou délinquantes ${ }^{6}$.

Deuxième facteur dont nous souhaitions contrôler les effets : la fréquence de leurs délits ou leur «degré d'activité». Les revenus criminels de chaque délinquant dépendent de ses lambdas. Il va de soi que plus les sujets commettent de délits contre les biens ou de transactions illicites, plus leurs revenus seront majorés (Tremblay et Morselli, 2000; McCarthy et Hagan, 2001). En incorporant les lambdas individuels comme variable indépendante, il en résulte que l'influence du capital social sur les revenus criminels individuels doit être interprétée en termes de «rendement» ou de «gains de capital»: le supplément de revenu illicite induit par chaque unité supplémentaire de capital social. La fréquence de base des délits varie selon la gravité des délits (on ne commet pas d'homicides au même rythme que des cambriolages) et selon le type de délinquance (délinquance de marché vs délinquance d'appropriation (Tremblay, 1999)). La délinquance de marché s'actualise à coups de transaction ponctuelle entre parties consentantes, qu'il s'agisse de contrebande, de revente de biens volés ou fraudés, de prêt usuraire,

6. Voir, par exemple, les travaux de Grasmick et al. (1993), Nagin et Paternoster (1993), Piquero et Tibbets (1996), Longshore et al. (1996), Sellers (1999), et Delisi et al. (2003). 
de proxénétisme ou de trafic de drogues illicites. La délinquance d'appropriation, quant à elle, est intrinsèquement antagonique et prend la forme d'attaques contre autrui ou contre leurs biens (cambriolages, vols qualifiés, fraudes, vols de véhicules, etc.). Les distributions des lambdas individuels étant très asymétriques (voir tableau 1), nous les avons également normalisées par transformation logarithmique (une stratégie utilisée également par Longshore et al., 1996 et Tremblay et Morselli, 2000). Les moyennes géométriques des données présentées dans le tableau 1 indiquent que les délinquants de cet échantillon avaient commis en moyenne 9 «attaques prédatrices» et participé à 91 transactions illicites au cours des 36 mois de la période d'analyse. Il est normal que les échanges illicites se produisent à une fréquence individuelle de base plus élevée que les prédations (une observation maintes fois constatée; voir par exemple Piquero et al., 2003). Compte tenu des dynamiques très différentes de ces deux formes de délinquance (voir à ce sujet Naylor, 2003), il était préférable d'analyser séparément la délinquance contre les biens et la délinquance de marché des sujets de l'échantillon. Tous ceux qui participaient aux marchés illicites ont été ainsi regroupés sous une même étiquette (la «délinquance de trafic » du tableau 2) quel que soit le nombre de vols ou de fraudes qu'ils avaient également commis. Tous ceux qui pratiquaient une délinquance d'appropriation ont également été regroupés sous une deuxième étiquette (la «délinquance contre les biens» du tableau 2), quelle que soit la fréquence des transactions illicites auxquelles ils avaient participé. Les deux regroupements se chevauchent partiellement, 31 sujets ou $20 \%$ de l'échantillon pratiquant les deux formes de délinquance.

Les trois autres variables dont nous voulions maintenir les effets constants ont été l'âge des sujets, leurs revenus licites et leurs coûts pénaux. Au début de la période d'analyse, les sujets avaient 28 ans en moyenne et les deux tiers d'entre eux entre 19 et 37 ans. Leurs revenus «légitimes» ne dépassaient pas les 25000 \$ pour l'ensemble de la période. Environ $14 \%$ seulement des sujets avaient évité l'incarcération durant cette période et, à l'autre extrême, $6 \%$ avaient passé les trois quarts de leur temps en prison: en moyenne, les délinquants interrogés avaient passé 1 jour sur 5 en prison durant les 36 mois qui avaient précédé leur incarcération actuelle. La durée présomptive moyenne des sentences qu'ils purgeaient était en moyenne de six ans. Bon nombre d'entre eux peuvent espérer être libérés sous condition au bout de trois ans. 
T A B LEA U 2

Analyses de régression multiple des revenus criminels d'un échantillon de détenus fédéraux

\begin{tabular}{|c|c|c|c|c|c|c|c|c|c|c|c|c|}
\hline \multirow[b]{2}{*}{ Variables } & \multicolumn{3}{|c|}{ Modèle 1} & \multicolumn{3}{|c|}{ Modèle 2} & \multicolumn{3}{|c|}{ Modèle 3} & \multicolumn{3}{|c|}{ Modèle 4} \\
\hline & $\begin{array}{c}\text { Tous les } \\
\text { délinquants }\end{array}$ & $\begin{array}{c}\text { Délinquance } \\
\text { de trafic }\end{array}$ & $\begin{array}{c}\text { Délinquance } \\
\text { contre } \\
\text { les biens }\end{array}$ & $\begin{array}{c}\text { Tous les } \\
\text { délinquants }\end{array}$ & $\begin{array}{c}\text { Délinquance } \\
\text { de trafic }\end{array}$ & $\begin{array}{c}\text { Délinquance } \\
\text { contre } \\
\text { les biens }\end{array}$ & $\begin{array}{l}\text { Tous les } \\
\text { délinquants }\end{array}$ & $\begin{array}{c}\text { Délinquance } \\
\text { de trafic }\end{array}$ & $\begin{array}{c}\text { Délinquance } \\
\text { contre } \\
\text { les biens }\end{array}$ & $\begin{array}{c}\text { Tous les } \\
\text { délinquants }\end{array}$ & $\begin{array}{c}\text { Délinquance } \\
\text { de trafic }\end{array}$ & $\begin{array}{c}\text { Délinquance } \\
\text { contre } \\
\text { les biens }\end{array}$ \\
\hline & $\begin{array}{l}\text { b (e.t.) } \\
\text { Bêta }\end{array}$ & $\begin{array}{l}\text { b (e.t.) } \\
\text { Bêta }\end{array}$ & $\begin{array}{l}\text { b (e.t.) } \\
\text { Bêta }\end{array}$ & $\begin{array}{l}\text { b (e.t.) } \\
\text { Bêta }\end{array}$ & $\begin{array}{l}\text { b (e.t.) } \\
\text { Bêta }\end{array}$ & $\begin{array}{l}\text { b (e.t.) } \\
\text { Bêta }\end{array}$ & $\begin{array}{l}\text { b (e.t.) } \\
\text { Bêta }\end{array}$ & $\begin{array}{l}\text { b (e.t.) } \\
\text { Bêta }\end{array}$ & $\begin{array}{l}\text { b (e.t.) } \\
\text { Bêta }\end{array}$ & $\begin{array}{l}\text { b (e.t.) } \\
\text { Bêta }\end{array}$ & $\begin{array}{l}\text { b (e.t.) } \\
\text { Bêta }\end{array}$ & $\begin{array}{l}\text { b (e.t.) } \\
\text { Bêta }\end{array}$ \\
\hline Âge & $\begin{array}{c}-, 032(, 014) \\
-, 163\end{array}$ & $\begin{array}{c}-, 026(, 014) \\
-, 164\end{array}$ & $\begin{array}{c}-, 019(, 018) \\
-, 098\end{array}$ & $\begin{array}{c}-, 025(, 014) \\
-, 125\end{array}$ & $\begin{array}{c}-, 017(, 014) \\
-, 108\end{array}$ & $\begin{array}{c}-, 017(, 018) \\
-, 087\end{array}$ & $\begin{array}{c}-, 018(, 014) \\
-, 092\end{array}$ & $\begin{array}{c}-, 016(, 014) \\
-, 098\end{array}$ & $\begin{array}{c}-, 002(, 018) \\
-, 011\end{array}$ & $\begin{array}{c}-, 016(, 014) \\
-, 083\end{array}$ & $\begin{array}{c}-, 016(, 014) \\
-, 098\end{array}$ & $\begin{array}{c}-, 006(, 019) \\
-, 029\end{array}$ \\
\hline $\begin{array}{l}\text { Revenus criminels } \\
\text { ajustés }\end{array}$ & $\begin{array}{c}-, 104(, 088) \\
-, 080\end{array}$ & $\begin{array}{c}-, 261(, 081) \\
-, 258 \\
\star *\end{array}$ & $\begin{array}{c}-, 070(, 107) \\
-, 058\end{array}$ & $\begin{array}{c}-, 148(, 089) \\
-, 114\end{array}$ & $\begin{array}{c}-, 299(, 082) \\
-, 295 \\
* * *\end{array}$ & $\begin{array}{c}-, 087(, 110) \\
-, 071\end{array}$ & $\begin{array}{c}-, 150(, 087) \\
-, 116\end{array}$ & $\begin{array}{c}-, 295(, 082) \\
-, 292 \\
* * *\end{array}$ & $\begin{array}{c}-, 108(, 107) \\
-, 089\end{array}$ & $\begin{array}{c}-, 147(, 086) \\
-, 113\end{array}$ & $\begin{array}{c}-, 284(, 081) \\
-, 281 \\
* * *\end{array}$ & $\begin{array}{c}-, 110(, 107) \\
-, 091\end{array}$ \\
\hline $\begin{array}{l}\text { Lambdas ajustés } \\
\text { (délits contre les biens) }\end{array}$ & $\begin{array}{c}, 438(, 113) \\
, 275 \\
* * *\end{array}$ & $\begin{array}{l}, 152(, 106) \\
, 123\end{array}$ & $\begin{array}{c}, 517(, 131) \\
, 348 \\
\star * \star\end{array}$ & $\begin{array}{c}, 440(, 111) \\
, 276 \\
\star * \star\end{array}$ & $\begin{array}{c}, 161(, 105) \\
, 130\end{array}$ & $\begin{array}{c}, 508(, 132) \\
, 342 \\
* * *\end{array}$ & $\begin{array}{c}, 346(, 117) \\
, 217 \\
\star \star\end{array}$ & $\begin{array}{c}, 128(, 112) \\
, 103\end{array}$ & $\begin{array}{c}, 450(, 130) \\
, 302 \\
* * *\end{array}$ & $\begin{array}{c}, 330(, 116) \\
, 207 \\
\star \star\end{array}$ & $\begin{array}{c}, 108(, 110) \\
, 087\end{array}$ & $\begin{array}{c}, 428(, 131) \\
, 288 \\
\star *\end{array}$ \\
\hline $\begin{array}{l}\text { Lambdas ajustés } \\
\text { (délits de trafic) }\end{array}$ & $\begin{array}{c}, 468(, 071) \\
, 456 \\
* \star *\end{array}$ & $\begin{array}{c}, 548(, 088) \\
, 505 \\
* * *\end{array}$ & $\begin{array}{c}, 354(, 079) \\
, 395 \\
\star * *\end{array}$ & $\begin{array}{c}, 426(, 071) \\
, 415 \\
\star \star *\end{array}$ & $\begin{array}{c}, 507(, 089) \\
, 467 \\
\star \star \star \\
\end{array}$ & $\begin{array}{c}, 337(, 083) \\
, 375 \\
* * * \\
\end{array}$ & $\begin{array}{c}, 366(, 075) \\
, 357 \\
\star \star \star\end{array}$ & $\begin{array}{c}, 471(, 099) \\
, 434 \\
\star * \star\end{array}$ & $\begin{array}{c}, 235(, 090) \\
, 263 \\
\star \star\end{array}$ & $\begin{array}{c}, 351(, 074) \\
, 343 \\
\star \star \star \\
\end{array}$ & $\begin{array}{c}, 449(, 097) \\
, 414 \\
\star \star *\end{array}$ & $\begin{array}{c}, 223(, 090) \\
, 248 \\
*\end{array}$ \\
\hline $\begin{array}{l}\text { Proportion de jours de } \\
\text { privation de liberté }\end{array}$ & $\begin{array}{l}, 405(, 496) \\
\quad, 056\end{array}$ & $\begin{array}{c}-, 074(, 472) \\
-, 013\end{array}$ & $\begin{array}{c}, 590(, 545) \\
\quad, 097\end{array}$ & $\begin{array}{c}, 350(, 488) \\
, 048\end{array}$ & $\begin{array}{c}-, 101(, 464) \\
-, 017\end{array}$ & $\begin{array}{c}, 598(, 547) \\
, 098\end{array}$ & $\begin{array}{c}, 132(, 490) \\
, 018\end{array}$ & $\begin{array}{c}-, 160(, 470) \\
-, 028\end{array}$ & $\begin{array}{c}123(, 563) \\
, 020\end{array}$ & $\begin{array}{c}, 092(, 484) \\
, 013\end{array}$ & $\begin{array}{c}-, 145(, 462) \\
-, 025\end{array}$ & $\begin{array}{c}, 039(, 566) \\
, 006\end{array}$ \\
\hline $\begin{array}{l}\text { Durée de la sentence } \\
\text { actuelle }\end{array}$ & $\begin{array}{c}, 001(, 001) \\
, 042\end{array}$ & $\begin{array}{c}, 001(, 001) \\
, 033\end{array}$ & $\begin{array}{c}-, 005(, 004) \\
-, 130\end{array}$ & $\begin{array}{c}, 001(, 001) \\
, 059\end{array}$ & $\begin{array}{c}, 001(, 001) \\
, 047\end{array}$ & $\begin{array}{c}-, 005(, 004) \\
-, 131 \\
\end{array}$ & $\begin{array}{c}, 001(, 001) \\
, 065\end{array}$ & $\begin{array}{c}, 001(, 001) \\
, 050\end{array}$ & $\begin{array}{c}-, 003(, 004) \\
-, 066\end{array}$ & $\begin{array}{c}, 001(, 001) \\
, 061\end{array}$ & $\begin{array}{c}, 001(, 001) \\
, 040\end{array}$ & $\begin{array}{c}-, 002(, 004) \\
-, 057\end{array}$ \\
\hline $\begin{array}{l}\text { Capital social des réseaux } \\
\text { personnels de } \\
\text { co-délinquants }\end{array}$ & - & - & - & $\begin{array}{c}, 117(, 047) \\
, 178 \\
*\end{array}$ & $\begin{array}{c}, 088(, 044) \\
, 178 \\
*\end{array}$ & $\begin{array}{c}, 044(, 062) \\
, 067\end{array}$ & $\begin{array}{c}110(, 047) \\
, 166 \\
*\end{array}$ & $\begin{array}{c}, 090(, 044) \\
, 181 \\
*\end{array}$ & $\begin{array}{c}, 046(, 060) \\
, 071\end{array}$ & $\begin{array}{c}, 115(, 046) \\
, 173 \\
*\end{array}$ & $\begin{array}{c}, 087(, 043) \\
, 176 \\
*\end{array}$ & $\begin{array}{c}, 062(, 061) \\
, 095\end{array}$ \\
\hline $\begin{array}{l}\text { Inaptitude à se contrôler } \\
\text { (low self control) }\end{array}$ & - & - & - & - & - & - & $\begin{array}{c}.019(, 008) \\
, 184 \\
\star\end{array}$ & $\begin{array}{c}, 007(, 008) \\
, 084\end{array}$ & $\begin{array}{c}, 027(, 011) \\
, 276 \\
*\end{array}$ & $\begin{array}{c}.018(, 008) \\
, 168 \\
*\end{array}$ & $\begin{array}{c}, 007(, 008) \\
, 090\end{array}$ & $\begin{array}{c}, 025(, 011) \\
, 263 \\
*\end{array}$ \\
\hline $\begin{array}{l}\text { Capital social } X \\
\text { inaptitude à se contrôler }\end{array}$ & - & - & - & - & - & - & - & - & - & $\begin{array}{c}-, 274(, 131) \\
-, 139 \\
*\end{array}$ & $\begin{array}{c}-, 229(, 112) \\
-, 160 \\
*\end{array}$ & $\begin{array}{c}-, 193(, 165) \\
-, 104\end{array}$ \\
\hline Constante & $\begin{array}{c}4,438 \\
(, 599) \\
\star \star \star\end{array}$ & $\begin{array}{c}4,908 \\
(, 593) \\
* * *\end{array}$ & $\begin{array}{c}4,257 \\
(, 736) \\
\star * \star\end{array}$ & $\begin{array}{c}4,001 \\
(, 615) \\
* * *\end{array}$ & $\begin{array}{c}4,534 \\
(, 612) \\
* * *\end{array}$ & $\begin{array}{c}4,134 \\
(, 758) \\
* * *\end{array}$ & $\begin{array}{c}2,568 \\
(, 861) \\
* \star\end{array}$ & $\begin{array}{c}4,075 \\
(, 814) \\
* * *\end{array}$ & $\begin{array}{c}1,840 \\
(1,168)\end{array}$ & $\begin{array}{c}2,738 \\
(, 855) \\
* *\end{array}$ & $\begin{array}{c}4,130 \\
(, 801) \\
\star * \star\end{array}$ & $\begin{array}{c}2,067 \\
(1,182)\end{array}$ \\
\hline $\mathrm{R}^{2}$ & 336 & ,398 & 361 &, 362 &, 423 & 365 &, 385 &, 428 &, 408 &, 404 &, 452 & ,417 \\
\hline
\end{tabular}

${ }^{*} p<0,05 ;{ }^{* *} p<0,01 ;{ }^{* * *} p<0,001 \quad\left(\mathrm{n}_{\text {tous délinquants }}=156\right) ;\left(\mathrm{n}_{\text {délinquants de marché }}=103\right) ;\left(\mathrm{n}_{\text {délinquants contre les personnes }}=97\right)$ 


\section{Âge, coûts et bénéfices}

La stratégie d'analyse procède en trois étapes. Le premier modèle d'analyse du tableau 2 incorpore l'ensemble des facteurs dont nous souhaitons contrôler statistiquement les effets ${ }^{7}$. Le deuxième modèle examine si le capital social des délinquants augmente leur performance. Les deux variantes que présentent les modèles 3 et 4 du tableau 2 évaluent la pertinence de l'hypothèse concurrente selon laquelle les prédispositions individuelles des délinquants (leur aptitude à contrôler leurs désirs, leurs humeurs ou leurs impulsions) expliqueraient en dernière analyse le succès relatif de leurs activités.

Depuis Quételet, on n'a cessé de faire état d'une relation inverse très marquée entre l'âge des délinquants et leur penchant au crime (Farrington, 1986; Sampson et Laub, 1993; Warr, 1998, 2002). Dans cette étude, ce qui est examiné n'est pas le penchant au crime (un individu s'abstient-il ou non de s'engager dans la délinquance?) mais la performance des délinquants - une dimension très différente de leur comportement. Ce qui se dégage du premier modèle d'analyse est que les revenus illicites des sujets diminuent en fonction de leur âge (coefficient de -0,032, p < 0,05), les jeunes adultes se montrant plus performants que les plus âgés (voir aussi en annexe la corrélation entre âge et revenus). Cette «prime à la jeunesse » se manifeste davantage (comme en témoigne la valeur du coefficient de régression) chez les délinquants qui s'investissent dans les trafics que chez les autres. On pourrait peutêtre en conclure que la carrière typique de bon nombre de délinquants connaîtrait une séquence particulièrement prometteuse dans la vingtaine ou la trentaine, mais qu'elle serait par la suite parsemée d'embûches. Mais il est possible aussi que les délinquants les plus performants se soient «retirés de la course » dès leurs premiers revers de fortune et que la performance des autres délinquants ait été passablement constante au fil du temps ou qu'elle se soit améliorée pour certains d'entre eux avec

7. Les analyses de régression multiple que nous utilisons dans le tableau 2 postulent que la réussite individuelle des délinquants dépend d'un ensemble de facteurs indépendants qui s'ajoutent les uns aux autres, et dont on souhaite évaluer l'influence directe et spécifique. Il s'agit d'une structure causale très simple qui ne prend pas en considération leur influence indirecte, la manière dont ces facteurs s'influencent mutuellement ou sont eux-mêmes influencés par la variable dépendante elle-même. En outre, $10 \%$ des délinquants de l'échantillon ne déclarent aucun revenu de leurs activités illicites, ce qui contrevient à la distribution qu'une analyse de régression requiert pour sa variable dépendante. Cette distorsion est cependant sans conséquence. On obtient des résultats analogues à ceux du tableau 2 lorsqu'on procède à des régressions de type Tobit. 
l'expérience. Il est difficile de se prononcer en l'absence de données longitudinales.

Parmi les autres facteurs dont nous voulions évaluer les effets collatéraux sur les revenus, on trouve les coûts pénaux auxquels s'exposent les délinquants. Plus leurs revenus sont importants, plus leur sentence actuelle et plus le nombre de jours passés en prison durant la fenêtre des 36 mois de la période d'analyse augmentent. Mais ni l'un ni l'autre ne sont statistiquement significatifs. Ce résultat est important parce qu'il indique que les coûts pénaux que les tribunaux et les agences de libération conditionnelle imposent aux délinquants ne sont pas proportionnels aux bénéfices qu'ils retirent de leurs activités. Un résultat déjà observé en ré-analysant les enquêtes de la Rand (Tremblay et Morselli, 2001; Robitaille, 2001), mais il est instructif que nous puissions le corroborer à nouveau sur la base d'un échantillon de détenus tout à fait différent.

Comme prévu, plus les délinquants sont actifs, plus leurs revenus augmentent. On notera que le degré d'activité des délinquants dépend de ce qu'ils font et non pas seulement de ce qu'ils sont: le tempo qui rythme les attaques varie de manière indépendante de celui qui régit leurs transactions illicites, ce qui nous amène à conclure que la fréquence de base avec laquelle les individus commettent des délits obéit à des contraintes objectives qui s'imposent aux délinquants (Tremblay, 1999). On observe aussi que pour les sujets dont la dominante est une délinquance de marché, seul le tempo de leurs transactions influence leurs revenus. Pour les sujets dont la dominante est une délinquance d'appropriation, le montant de leurs revenus illicites dépend à la fois de la fréquence des délits contre les biens qu'ils commettent et du volume des transactions illicites auxquelles ils participent. Il semble bien qu'il soit plus aisé de s'enrichir en trafiquant qu'en volant (les corrélations présentées en annexe sont respectivement de 0,44 et de 0,27).

Plus les délinquants retirent des revenus appréciables de leurs activités illicites, moins ils devraient être enclins à investir le marché conventionnel du travail. Et vice versa: moins les individus réussissent à trouver un emploi satisfaisant, plus ils devraient se laisser tenter par le crime. Dans les deux cas, les revenus illicites devraient varier en fonction inverse des revenus légitimes. C'est effectivement ce qu'ont observé Viscusi (1986) d'une part, et Uggen et Thompson (2003), d'autre part. Mais on peut également penser que les individus talentueux devraient manifester leurs talents dans diverses sphères d'activités et que ceux 
qui réussissent mieux que d'autres à tirer des avantages appréciables de leurs activités illicites devraient atteindre des résultats similaires lorsqu'ils acceptent de s'investir dans des activités «conventionnelles». Celui qui brille par ses talents de revendeur de cocaïne, devrait briller avec le même éclat dans la vente d'autres produits. Plusieurs études, en tous cas, font état d'une corrélation positive entre les revenus légitimes et illégitimes des délinquants (Reuter et al., 1990 ; Tremblay et Morselli, 2000; Robitaille, 2001). Les résultats du tableau 2 suggèrent, sous réserve d'analyses plus approfondies, que la première hypothèse semble plus probante que la deuxième: plus les sujets ont un parcours délinquant performant, moins ils sont enclins à s'investir dans le marché du travail. Et réciproquement. Mais cela est vrai seulement pour ceux qui optent pour la délinquance des trafics (coefficient de -0,261, p $<0,01$ du modèle 1 du tableau 2). On ne retrouve pas la même dynamique chez les délinquants qui privilégient une délinquance d'appropriation (coefficient de $-0,065$, n.s.), probablement parce que leurs revenus sont plus modestes et ne les autorisent pas à déserter le marché de travail.

\section{Capital social et performance délinquante}

Le deuxième modèle du tableau 2 examine l'argument selon lequel, toutes choses étant égales par ailleurs, les délinquants sont d'autant plus performants que leurs réseaux de co-délinquance sont riches en capital social. Ce capital n'est pas, remarquons-le, un attribut strictement individuel mais une propriété qui émerge du réseau lui-même et de la position relative que les sujets y occupent. Le résultat principal qui se dégage du modèle 2 est que, toutes choses étant égales par ailleurs, plus leurs réseaux sont riches en capital social, plus les délinquants en font bon usage et plus leurs revenus illicites augmentent (coefficient de régression de $+0,178, p<0,05$ ). On ne peut exclure que la relation causale s'exerce en sens contraire et que la réussite économique contribue elle aussi à accroître le capital social des délinquants. L'efficacité de cette «capitalisation» se manifeste quels que soient l'âge des sujets, leurs revenus conventionnels ou le nombre de délits qu'ils commettent. Il est instructif que cet impact soit beaucoup plus marqué lorsqu'il s'agit de délinquants qui tirent leurs revenus de trafics illicites plutôt que de délits contre les biens (coefficients de 0,178 et de 0,067 respectivement). On peut penser que la délinquance 
de trafic est gouvernée, sous peine de faillite ou d'élimination rapide, par les mêmes impératifs et les mêmes contraintes que toutes les autres activités commerciales et il n'est pas surprenant, de ce point de vue, que délinquants, employés et commerçants doivent adopter des stratégies similaires pour atteindre la réussite. On notera qu'en incorporant le capital social des délinquants dans le modèle d'analyse, le déclin des revenus en fonction de l'âge cesse d'être statistiquement significatif. On doit en conclure que les délinquants dans la vingtaine ou la trentaine bénéficient d'un capital social plus riche que ceux qui sont dans la quarantaine ou la cinquantaine. Le capital social de chaque délinquant n'est pas sous un contrôle exclusif et, contrairement aux lois habituelles qui gouvernent la gestion des fortunes, il n'existe aucune raison particulière pour que ce capital social se renouvelle de lui-même ou qu'il augmente naturellement. On peut penser au contraire, tout particulièrement lorsqu'il s'agit des délinquants, que ce capital social tendra à se dégrader avec le temps, notamment en raison des forces considérables qui concourent à déstabiliser les réseaux de co-délinquance (incarcération, mort, exil et trahison des uns et des autres, dommages collatéraux imposés aux enfants et aux conjoints) et de l'absence d'institutions pour pacifier les différends ou assurer une prévisibilité et une continuité aux échanges. Il resterait à préciser, mais cela déborde le cadre de cette étude, si les délinquants les plus avantagés en capital social l'ont «hérité» d'autres délinquants (de leurs mentors, par exemple) ou si, au contraire, ils sont parvenus à le «développer» de quelque façon (sur ce thème, voir les études de cas de Morselli, 2001; 2003).

Comme on l'a déjà souligné, on ne peut pas exclure d'office l'hypothèse selon laquelle le capital social des délinquants serait l'épiphénomène d'un trait de personnalité qui serait à l'origine à la fois de leur performance et de leur capital social. Pour évaluer les mérites de ce contre-argument, nous avons ajouté aux variables des deux premiers modèles d'analyse une échelle qui mesure le degré de présentisme ou d'impulsivité des sujets (modèle 3 du tableau 2). S'il est vrai que les délinquants les plus performants sont les moins impulsifs et que ce trait de personnalité nuit à leur capital social (en faisant fuir les co-délinquants les «plus utiles»), on devrait admettre que l'interprétation selon laquelle celui-ci déterminerait leur performance serait fallacieuse. Les résultats du troisième modèle d'analyse sont cependant très clairs: l'effet observé du capital social sur le rendement des activités de trafic des délinquants (le coefficient de 0,178 du modèle 2) est direct et ne dépend pas du 
degré d'impulsivité des sujets (ce même coefficient a à peu près la même valeur - 0,166 - dans le modèle 3). En revanche, et de manière quelque peu étonnante, ce que les criminologues qualifient, non sans condescendance, de «manque de contrôle de soi» ne constitue pas un handicap lorsqu'il s'agit de réussite délinquante.

Nos analyses indiquent en effet que plus les délinquants sont «impulsifs», présentistes ou «imprudents», plus les revenus qu'ils retirent de chaque délit qu'ils commettent ou de chaque transaction illicite à laquelle ils participent augmentent (coefficient de $+0,184$, $p<0,05$ ). Cet effet est très marqué pour le groupe de délinquants qui privilégient une délinquance d'appropriation (coefficient de $+0,276, p<0,05$ ), mais ne l'est pas pour ceux qui s'investissent dans une délinquance de marché. Mais même dans ce dernier cas, une prédisposition à l'impulsivité a des effets «positifs» sur leurs revenus (coefficients de $+0,084$ dans le modèle 3 et de $+0,09$ dans le modèle 4 du tableau 2). Autrement dit, un défaut personnel - un manque de contrôle de soi - se traduit par un bénéfice - des revenus illicites plus élevés sur une période qui s'étale tout de même sur trois ans. Quoiqu'il était inattendu, ce résultat est-il en lui-même bizarre? Nous pensons que non. Les revenus que les individus retirent de leurs activités illégales les dédommagent des risques qu'ils courent (Viscusi, 1986; Reuter et al., 1990). McCarthy et Hagan (2001) notent que le goût du risque combiné à leur compétence naturelle a pour effet d'augmenter les revenus illicites individuels des délinquants juvéniles qu'ils ont étudiés. Pour s'engager dans la délinquance, les individus doivent prendre des risques et ceux qui «aiment» en prendre devraient être avantagés au départ. Si les délinquants ne prenaient pas des risques importants, ils ne le seraient sans doute jamais devenus. Il reste que l'essentiel de notre propos n'est pas de comparer les délinquants à ceux qui ne le sont pas, mais de comparer les délinquants entre eux. Certains délinquants sont plus téméraires que d'autres, prennent davantage de risques que d'autres et acceptent des paris que d'autres délinquants refuseraient de prendre. S'agit-il d'un handicap? Les résultats disent que non et ils donnent plutôt raison aux délinquants qui ont du cran, qui saisissent rapidement l'occasion qui passe et qui se fient à leur «flair».

Il reste que les gains en rendement de cette témérité sont beaucoup plus nets pour la délinquance d'appropriation que pour la délinquance de trafic et que les revenus du premier groupe sont moins élevés que ceux du deuxième (les constantes des équations de régression pour 
chaque groupe en témoignent). Il est possible que la témérité soit avantageuse lorsqu'il s'agit de revenus modestes, mais qu'elle cesse de l'être au-delà d'un certain seuil. On aurait pu s'attendre cependant à ce que l'impulsivité des sujets nuise à la conservation de leur capital social ou $\mathrm{du}$ moins qu'elle devienne un handicap dans le contexte beaucoup plus organisé de la délinquance de trafic. Mais les résultats sont très clairs: le manque de contrôle sur soi ne nuit ni à leurs revenus (la corrélation indiquée en annexe est de $+0,44$ ) ni à leur capital social (la corrélation est de $+0,31$ ). Il est possible néanmoins que l'effet de turbulence attendu ne s'exerce pas directement mais conditionnellement. Autrement dit, il est possible qu'une trop grande témérité ne nuise ni au capital social des délinquants ni à leurs revenus considérés séparément, mais entame la force de la relation entre ces deux termes et diminue la capacité des délinquants de traduire en espèces sonnantes et trébuchantes les occasions que leur offre leur capital social. Même si A et B ont des effets positifs sur $\mathrm{C}$, il est possible que la présence de A fasse en sorte que la relation entre $\mathrm{B}$ et $\mathrm{C}$ soit moins forte qu'elle ne le serait en son absence. De tels effets indirects sont souvent qualifiés d'effets d'interaction ou d'effets conditionnels. Le dernier modèle du tableau 2 propose de vérifier si l'impact du capital social des sujets sur leurs revenus dépend de leur degré d'impulsivité et incorpore une variable d'interaction dont les deux termes (capital social et faible contrôle de soi) ont été préalablement standardisés. Les résultats sont plutôt concluants: tous les délinquants retirent un avantage tangible d'un supplément de capital social, mais plus les délinquants sont impulsifs, moins cet avantage est important: le coefficient du terme d'interaction est négatif et significatif $(-0,274, p<0,05$, modèle 4). Autrement dit, leurs revenus augmentent quand même, mais ils augmentent "plus lentement». Cet effet d'interaction régit principalement la délinquance de trafic (coefficient de $-0,229$, $p<0,05$ ), même si on le retrouve de manière latente dans la délinquance d'appropriation (coefficient de $-0,193$, n.s.). En matière de trafics illicites, les délinquants trop impulsifs seraient moins performants qu'ils pourraient l'être, et cette baisse « relative» de performance se manifestera avec d'autant plus d'acuité que leurs revenus seront élevés ou que leur capital social sera étendu. 


\section{Conclusion}

S'appuyant sur les résultats d'une enquête de terrain auprès d'un échantillon autoréférentiel de cambrioleurs, Wright et Decker (1977:4) en arrivent à la conclusion que les délinquants « ratés» sont surreprésentés dans les enquêtes auprès de détenus. Il reste que le sondage dont nous venons ici de présenter quelques résultats indique assez nettement que l'on trouve en prison une diversité appréciable de performance : autant les revenus qu'ils avaient retirés de leurs activités illégales durant les 36 mois précédant leur incarcération pouvaient être qualifiés de médiocres pour certains, autant ils étaient appréciables et dignes de mention pour d'autres. C'est également la conclusion à laquelle parviennent d'autres enquêtes similaires auprès de délinquants juvéniles ou adultes, incarcérés ou en liberté (Viscusi, 1986; Reuter et al., 1990; Matsueda et al., 1992; Tremblay et Morselli, 2000 ; McCarthy et Hagan, 2001 ; Robitaille, 2001). D'autres auteurs concèdent que les détenus puissent constituer un échantillon représentatif de délinquants persistants, mais récusent cependant d'accorder une validité quelconque aux témoignages de réussite, préférant y voir le signe d'une propension à l'esbroufe et à la rationalisation (Wilson et Abrahamse, 1992). Mais ce mépris condescendant ne s'appuie en réalité sur aucune base solide de recherches empiriques: la cohérence qui se dégage du croisement des réponses que les détenus ont données à un ensemble de questions relatives à leur performance, la lucidité de l'appréciation des délinquants de leurs succès ou de leurs échecs relatifs, les propositions parfaitement conséquentes qu'on peut en dériver dès lors qu'on leur accorde le bénéfice du doute, tout cela fait en sorte qu'il est difficile de croire que les détenus qui ont participé à cette étude, et qui n'avaient rien à gagner à cette coopération, se soient donné la peine d'anticiper nos hypothèses de recherche et de maintenir la cohérence d'un récit imaginaire tout au long du questionnaire, alors qu'il leur eût été si facile de décliner l'offre d'y répondre. On ne peut, en outre, à la fois admettre le bien-fondé des sondages de délinquance révélée qu'exploite massivement la criminologie contemporaine et récuser, du même souffle, les résultats des enquêtes dans lesquelles on demande aux sujets de nous indiquer les résultats ou les avantages qu'ils retirent de leur comportement délinquant. De plus, plusieurs des études que nous venons de citer n'ont pas manqué de proposer aux sujets d'estimer de différentes façons leurs revenus illicites pour évaluer le degré de convergence et de fiabilité de leurs déclarations. Or, 
les discordances observées n'ont rien d'excessives et semblent davantage imputables à la difficulté intrinsèque de la tâche qui leur est demandée qu'à d'autres facteurs adventices (Charest, 2004). En procédant par ailleurs à une transformation logarithmique de leurs revenus déclarés, on introduit d'office une correction à la baisse de leurs gains.

L'hypothèse de recherche que nous souhaitions mettre à l'épreuve dans cet article stipulait que le capital social des délinquants aurait un effet direct et positif sur leurs chances de réussir leur «carrière criminelle», ou du moins sur leur capacité d'augmenter les revenus qu'ils retirent de leurs activités illégales. Même si les réseaux de co-délinquance de certains sujets «ont» davantage de capital social, cette ressource n'est pas un attribut qu'ils possèdent à titre individuel mais un atout du réseau lui-même dont ils peuvent profiter à différents degrés selon la position qu'ils y occupent. En faisant la démonstration de cette hypothèse, il est apparu qu'un certain nombre de propositions couramment admises en sociologie criminelle mériteraient d'être revues et corrigées.

Tout d'abord, il ne semble pas que l'on gagne en rigueur en réduisant les opportunités criminelles aux occasions qui résultent de la convergence spatio-temporelle d'une cible, d'une victime potentielle et d'un délinquant motivé (Felson, 1998). Les opportunités décisives qui orientent les trajectoires délinquantes individuelles dépendent plutôt de la richesse des réseaux de co-délinquance qui s'y entrecroisent. Greenberg, Tamareli et Kelley (2002) rapportent que les délinquants qui bénéficient d'affiliations multiples peuvent "saisir» des occasions qui échappent aux délinquants qui se limitent au rayon d'action de leur clique. Ce résultat corrobore bien entendu la thèse générale selon laquelle moins les réseaux de co-délinquance sont redondants, plus ils sont riches en capital social, et plus les revenus que chacun en retire deviennent appréciables. On devrait se garder d'interpréter ce capital social en termes "volontaristes »: les individus n'ont pas de capital social au sens où ils ont un capital financier ou patrimonial; ils ne peuvent pas l'investir ou le gérer comme un portefeuille d'actions; ils peuvent néanmoins en faire un bon ou un mauvais usage, mais cet usage dépend en même temps aussi de celui qu'en font les autres membres, lointains ou proches, de leur réseau de relations. C'est pour cette raison que les délinquants ont de la difficulté à «conserver» leur capital social: non seulement ils n'en ont pas le contrôle, mais son émergence se heurte à un environnement hostile qui déploie des efforts constants et importants pour qu'il se dissipe. Une 
des limites de cette étude est d'avoir proposé une analyse qui était seulement transversale et non longitudinale du capital social des délinquants.

Certains estiment qu'une théorie générale du comportement délinquant n'est «réaliste» qu'à la condition expresse qu'elle le décrive comme une conduite essentiellement impulsive, polymorphe et qui ne procure aucun bénéfice durable ou appréciable à ses acteurs (Gottfredson et Hirschi, 1990). Cette thèse fait problème. En adoptant le délit ou le passage à l'acte comme unité d'analyse, on exclut d'office la séquence des délits successifs dans laquelle tel ou tel délit s'insère. On ne comprendra guère les réseaux de fraudeurs qui opèrent en volume en soulignant que les bénéfices que leur procure chaque transaction frauduleuse sont médiocres. Elle fait également problème à un autre point de vue: elle escamote les différences individuelles de performance délinquante et suppose que les occasions criminelles relèvent du hasard ou qu'elles ne peuvent être capitalisées. L'argument selon lequel un défaut de caractère ou un trait de personnalité (impulsivité, témérité, présentisme) expliquerait un défaut de résultat (les gains éphémères et médiocres de la délinquance) n'est pas particulièrement éclairant. La délinquance est par définition une activité à « haut risque» et les délinquants qui ne sont pas prêts à prendre des risques devraient en toute logique réussir moins bien dans cette voie que ceux qui «aiment»le risque. En décrivant les délinquants comme inaptes à "se contrôler», Gottfredson et Hirschi décrivent un état de choses qu'ils n'expliquent pas pour autant. Ils ne peuvent expliquer que la relation observée (impulsivité $=$ revenus criminels appréciables) contredise la congruence attendue (impulsivité $=$ revenus criminels médiocres). Comme ils conçoivent la délinquance, la toxicomanie, l'imprudence comme autant de manifestations équivalentes d'une même prédisposition générale, ils ne peuvent pas expliquer non plus que cette impulsivité puisse être un atout manifeste pour certaines activités illicites (la délinquance d'appropriation), mais une contrainte au deuxième degré pour d'autres (la délinquance de trafic) : à capital social constant, les délinquants les plus impulsifs ont, lorsqu'il s'agit de délits de trafic, plus de difficulté à obtenir le même rendement de ce capital. Les marchés illicites sont régis par des contraintes qui ne sont pas fondamentalement différentes de celles qui gouvernent les marchés licites et il n'est pas étonnant qu'ils favorisent les entrepreneurs les moins anomiques. Cette observation ne peut découler de la théorie "générale» du comportement délinquant 
que proposent Goffredson et Hirschi (1990), puisque celle-ci n'admet pas au départ que les délinquants puissent disposer d'un capital social de quelque valeur, qu'elle n'a rien à dire sur ses origines ou son impact sur les trajectoires individuelles.

Les données de cette étude indiquent que plus les revenus des délinquants sont élevés, notamment en matière de délinquance de trafic, moins ils se donnent la peine de participer au marché conventionnel $\mathrm{du}$ travail. Ils n'en ont probablement ni le temps ni la motivation, et leurs qualifications professionnelles ou scolaires sont généralement insuffisantes pour qu'ils puissent accéder à des positions qui leur procurent les mêmes avantages tangibles. En outre, le capital social et humain qu'ils acquièrent tout au long de leur trajectoire de délinquance n'est pas «transférable» au marché du travail qui exige, de manière générale, une attestation officielle des qualifications des candidats à l'emploi. La «réintégration» dans le marché du travail implique que les plus performants des délinquants devront se résigner à un déclassement ou à une baisse sévère de leurs revenus. Nos résultats rejoignent les conclusions auxquelles sont parvenues les recherches évaluatives sur les programmes de réinsertion professionnelle des ex-détenus: le problème principal réside dans le fait que les incitatifs financiers susceptibles de les motiver à «retourner» sur le marché du travail sont souvent inexistants ou dérisoires (Uggen, 2000; Uggen et Thompson, 2003). Il semble que ces programmes soient d'autant plus «efficaces» qu'ils s'adressent à une «clientèle» déjà acquise: les délinquants qui n'ont jamais déserté le marché du travail; ceux qui n'ont jamais réussi à retirer des revenus satisfaisants de leurs activités illicites et pour lesquels les coûts d'opportunité de réintégration sociale sont faibles; et finalement ceux qui ont perdu ou dissipé le capital social à l'origine de leurs épisodes de réussite. Les délinquants ne sont pas les seuls, cependant, à connaître des épisodes de contre-mobilité. Bon nombre d'individus connaissent «des parcours de mobilité à phases multiples, ou de mobilité suivie de contremobilité, qui peuvent paraître dans les enquêtes soit en mobilité ascendante, soit en mobilité descendante, alors qu'à terme ils aboutissent à une position de stabilité» (Merllié, 1994: 68; voir aussi Girod, 1971: 44-45). Lorsque les sociologues se sont intéressés à la récidive, ils ont été enclins à l'expliquer en raison des opportunités restreintes peu attrayantes qu'offre le marché du travail aux délinquants qui sortent de prison. Cette explication est bancale parce que la plupart des délinquants sont sous-qualifiés, alors que la plupart des trajectoires délinquantes sont 
relativement courtes et que les abandons de carrière sont prédominants (Tremblay, 1999). Elle cesserait de l'être si elle admettait que l'effet stipulé est conditionnel aux gains qu'ils retirent de leur délinquance. Lorsque leurs revenus illicites sont médiocres ou en chute libre, les délinquants se résignent à se «ranger»; lorsque leurs revenus sont appréciables ou supérieurs aux revenus d'emploi que leur offre le marché du travail, ils persistent. Mais ces revenus illicites sont intrinsèquement incertains et dépendent notamment de l'aptitude des délinquants à conserver, diversifier ou renouveler le capital social de leurs réseaux de co-délinquance. Il est possible que ce soit la volatilité de cette ressource qui explique pourquoi tant de délinquants finissent par se ranger. Une limite de cette étude est d'avoir analysé le capital social des délinquants comme une constante ou une ressource fixe. En réalité, il conviendrait de l'analyser comme un processus probablement «autodégradable».

Piliavin et al. (1986) ont montré que les choix des délinquants étaient davantage influencés par les bénéfices attendus de leurs activités illicites que par les coûts pénaux auxquels ils s'exposaient. On en a conclu que la rationalité des délinquants était «limitée», qu'ils souffraient de myopie temporelle ("présentisme») et que les avantages immédiats de leur délinquance pèseraient plus lourd dans leurs décisions que le cumul différé de ses désavantages. Rien n'est moins sûr. Nous n'avons observé dans cette étude aucun rapport direct entre la sévérité des sentences que purgeaient les détenus interrogés et leur réussite économique $(r=0,004)$. La réanalyse d'une enquête auprès de détenus américains, tel que le propose Robitaille (2001), montre que même si les délinquants les plus performants purgent des sentences plus longues, le temps supplémentaire qu'ils passaient en prison serait largement inférieur aux revenus supplémentaires qu'ils auraient retirés de leurs délits et qu'en bout de ligne, ce sont les délinquants qui déclarent le moins de revenus qui sont, en termes relatifs, les plus pénalisés. Il va de soi que le prix que les délinquants sont prêts à payer de leur personne (arrestations et condamnations) dépend des avantages qu'ils retirent de leurs délits une proposition que chacun tiendra pour banale, mais qui n'a jamais été scrutée en détail dans les recherches qui se proposent de donner une base empirique à la doctrine de la dissuasion. On doit reconnaître à Stafford et Warr (1993) le mérite d'intégrer les notions de dissuasion et d'opportunités criminelles: les individus apprécieraient les risques auxquels ils s'exposent, d'une part, en puisant dans le réservoir des situations dans lesquelles des membres de leur entourage (dissuasion 
générale) ou eux-mêmes (dissuasion spécifique) ont été arrêtés ou sanctionnés et, d'autre part, en tirant les leçons des expériences, au demeurant beaucoup plus fréquentes, des situations où les délits des autres (opportunités générales) ou les leurs propres (opportunités spécifiques) demeurent impunis. Un délit impuni étant un délit réussi, les «expériences d'impunité » auxquelles se réferent Stafford et Warr nous renvoient, tout compte fait, à leurs gains. Ce qui nous amène à conclure que l'appréciation des délinquants de leurs propres réussites et des réussites des autres délinquants de leur entourage (expériences d'impunité) devrait relativiser ou dramatiser, selon le cas, l'impact de leurs propres revers ou des échecs essuyés par les membres proches ou lointains de leurs réseaux de co-délinquance (expériences pénales).

\section{Références}

Adler, P.A. (1993 [1985]). Wheeling and dealing: ethnography of an upper-level drug dealing and smuggling community, $2^{\text {nd }}$ edition. New York: Columbia University Press.

Agnew, R. (1991). The interactive effect of peer variables on delinquency. Criminology, 29, 47-72.

Agnew, R. (1994). Delinquency and the desire for money. Justice Quarterly, 11, 411-427.

Albini, J.L. (1971). The american mafia: genesis of a legend. New York: Meredith.

Argyle, M. (1991). Cooperation: the basis of sociability. New York: Routledge.

Becker, G.S. (1993 [1964]). Human capital: a theoretical and empirical analysis, $3^{\text {rd }}$ edition. Chicago: University of Chicago Press.

Block, A., \& Chambliss, W.J. (1981). Organizing crime. New York : Elsevier.

Blok, A. (1974). The mafia of a sicilian village, 1860-1960: a study of violent peasant entrepreneurs. New York: Harper and Row.

Blumstein, A., Cohen, J., Roth, J.A., \& Visher, C.A. (1986). Criminal careers and «career criminals». Washington D.C. : National Academy Press.

Borgatti, S.P., Everett, M.G., \& Freeman, L.C. (1999). UCINET 5.0, Version 1.00. Natick: Analytic Technologies.

Boudon, R. (1973). L'inégalité des chances: la mobilité sociale dans les sociétés industrielles. Paris: Armand Colin.

Breiger, R.L. (1990). Social mobility and social structure. Cambridge: Cambridge University Press.

Burt, R.S. (1992). Structural holes: the social structure of competition. Cambridge: Harvard University Press.

Burt, R.S. (2001). Structural holes versus network closure as social capital. In N. Lin, K.Cook \& R.S. Burt (eds), Social capital: theory and research (31-56). New York: Aldine de Gruyter. 
Burt, R.S. (2002). The social capital of structural holes. In M. Guillén, R. Collins, P. England \& M. Meyer (eds), The new economic sociology: developments in an emerging field (148-190). New York: Russell Sage Foundation.

Chaiken, J.M., \& Chaiken, M.R. (1982). Varieties of criminal behavior. Santa Monica: Rand Corporation.

Charest, M. (2004). Peut-on se fier aux délinquants pour estimer leurs revenus criminels? Criminologie, 37 (2), 63-87.

Clausen, J.S. (1991). Adolescent competence and the shaping of the life course. American Journal of Sociology, 96, 805-842.

Cullen, F.T., \& Link, B.G. (1980). Crime as an occupation. Criminology, 18, 399410.

Cusson, M. (1981). Délinquants pourquoi? Montréal: Hurtubise.

Cusson, M. (1986). L'analyse stratégique et quelques développements en criminologie. Criminologie, 19, 53-72.

Delisi, M., Hochstetler, A., \& Murphy, D.S. (2003). Self-control behind bars : a validation study of the Grasmick et al. Scale. Justice Quarterly, 20, 241-263.

Duncan, G.J., \& Hoffman, S.D. (1981). The incidence and wage effects of overeducation. Economics of Education Review, 1, 75-86.

Érasme (1987 [1511]). Éloge de la folie, suivi de la Lettre d'Érasme à Dorpius. Paris: GF Flammarion.

Evans, D.T., Cullen, F.T., Burton Jr., V.S., Dunaway, R.G., \& Benson, M.L. (1997). The social consequence of self-control: testing the general theory of crime. Criminology, 35, 475-504.

Felson M. (1998). Crime and everyday life. Thousand Oaks: Pine Forge Press.

Farrington, D.F. (1986). Age and crime. In M. Tonry \& N. Morris (eds), Crime and Justice, vol. 7 (189-250). Chicago: Chicago University Press.

Gottfredson, M.R., \& Hirschi, T. (1990). A general theory of crime. Stanford: Standford University Press.

Girod, R. (1971). Mobilité sociale. Genève: Librairie Droz.

Granovetter, M. (1974). Getting a job: a study of contacts and careers. Cambridge: Harvard University Press.

Granovetter, M. (1985). Economic action and social structure: the problem of embeddedness. American Journal of Sociology, 91, 481-510.

Grasmick, H.S., Tittle, C.R., Bursik Jr., R.J., \& Arneklev, B.J. (1993). Testing the core empirical implications of Gottfredson and Hirschi's general theory of crime. Journal of Research in Crime and Delinquency, 30, 5-29.

Greenberg, D.F., Tamarelli, R., \& Kelley, M.S. (2002). The generality of the self-control theory of crime. In E. Waring \& D. Weisburd (eds), Crime and social organization. Advances in criminological theory series, vol. 10 (49-94). New Brunswick: Transaction.

Hagan, J. (1994). Crime and disrepute. Toronto: Pine Forge Press.

Hagan, J., \& McCarthy, B. (1997). Mean streets : youth crime and bomelessness. New York: Cambridge University Press. 
Haller, M.H. (1990). Illegal enterprise : a theoretical and historical interpretation. Criminology, 28, 207-235.

Haller, M.H. (1991). Life under Bruno: the economics of an organized crime family. Pennsylvania Crime Commission.

Hess, H. (1998 [1973]). Mafia and mafiosi: origin, power and myth. New York: New York University Press.

Ianni, F.J. (1972). A family business. New York: Russell Sage Foundation.

Jencks, C. (1979). Who gets abead? The determinants of economic success in America. New York: Basic Books.

Keane, C., Maxim, P.S., \& Teevan, J.J. (1993). Drinking and driving, self-control, and gender. Journal of Research in Crime and Delinquency, 30, 30-46.

Kleemans, E.R., \& van de Bunt, H.G. (1999). The social embeddedness of organized crime. Transnational Organized Crime, 5, 19-36.

Klerks, P. (2001). The network paradigm applied to criminal organizations. Connections, 24, 53-65.

Lemieux, V. (1999). Les réseaux d'acteurs sociaux. Paris: Presses Universitaires de France.

Levitt, S.D., \& Venkatesh, S.A. (2000). An economic analysis of a drug-selling gang's finances. Quarterly Journal of Economics, 115, 755-789.

Lin, N. (2001). Social capital: a theory of social structure and action. Cambridge: Cambridge University Press.

Longshore, D., Turner, S., \& Stein. J.A. (1996). Self-control in a criminal sample: an examination of construct validity. Criminology, 34, 209-228.

Matsueda, R.L., Gartner, R., Piliavin, I., \& Polakowski, M. (1992). The prestige of criminal and conventional occupations: a subcultural model of criminal activity. American Sociological Review, 57, 752-770.

McCarthy, B. (1996). The attitudes and actions of others: tutelage and Sutherland's theory of differential association. British Journal of Criminology, 36, 135-147.

McCarthy, B. (2002). New economics of sociological criminology. Annual Review of Sociology, 28, 417-442.

McCarthy, B., \& Hagan, J. (2001). When crime pays: capital, competence, and criminal success. Social Forces, 79, 1035-1059.

McCarthy, B., Hagan, J., \& Cohen, L.E. (1998). Uncertainty, cooperation, and crime: understanding the decision to co-offend. Social Forces, 77, 155-176.

Merllié, D. (1994). Les enquêtes de mobilité sociale. Paris: Presses Universitaires de France.

Morselli, C. (2001). Structuring Mr. Nice: entrepreneurial opportunities and brokerage positioning in the cannabis trade. Crime, Law, and Social Change, 35 (4), 203-244.

Morselli, C. (2003). Career opportunities and network-based privileges in the Cosa Nostra. Crime, Law, and Social Change, 39, 383-418.

Nagin, D.S., \& Paternoster, R. (1993). Enduring individual differences and rational choice theories of crime. Law and Society Review, 27, 467-496. 
Naylor, R.T. (2003). Towards a general theory of profit-driven crimes. British Journal of Criminology, 43, 81-101.

Paternoster, R., \& Piquero, A. (1995). Reconceptualizing deterrence: an empirical test of personal and vicarious experiences. Journal of Research in Crime and Delinquency, 32, 251-286.

Peterson, M.A., \& Braiker, H.B. (1981). Who commits crime: a survey of prison inmates. Cambridge: Oelgeschlager, Gunn and Hann.

Piliavin, I., Gartner, R., Thornton, C., \& Matsueda, R. (1986). Crime, deterrence, and rational choice. American Sociological Review, 51, 101-119.

Piquero, A., \& Pogarsky, G. (2002). Beyond Stafford and Warr's reconceptualization of deterrence : personal and vicarious experiences, impulsivity, and offending behavior. Journal of Research in Crime and Delinquency, 39, 153-186.

Piquero, A., \& Tibbetts, S. (1996). Specifying the direct and indirect effects of low-self control and situational factors in offenders' decision making: toward a more complete model of rational offending. Justice Quarterly, 13, 481-510.

Reuter, P., MacCoun, R., \& Murphy, P. (1990). Money from crime: a study of the economics of drug-dealing in Washington D.C.. Santa Monica: Rand.

Robitaille, C. (2001). Gains criminals et facteurs individuals de réussite: une ré-analyse du sondage de 1978 de la Rand Corporation. Mémore de maîtrise, École de Criminologie, Université de Montréal.

Sampson, R.J., \& Laub, J.H. (1993). Crime in the making: pathways and turning points through life. Cambridge: Harvard University Press.

Sellers, C.S. (1999). Self-control and intimate violence: an examination of the scope and specification of the general theory of crime. Criminology, 37, 375404.

Stafford, M.C., \& Warr, M. (1993). A reconceptualization of general and specific deterrence. Journal of Research in Crime and Delinquency, 30, 123-135.

Tremblay, P. (1993). Searching for suitable co-offenders. In R.V. Clarke \& M. Felson (eds), Routine activity and rational choice: advances in criminological theory. New Brunswick: Transaction.

Tremblay, P. (1999). Attrition, récidive et adaptation. Revue internationale de criminologie et de police technique et scientifique, 52, 163-178.

Tremblay, P., \& Morselli, C. (2000). Patterns in criminal achievement: Wilson and Abrahamse revisited. Criminology, 38, 633-659.

Uggen, C. (2000). Work as a turning point in the life course of criminals : a duration model of age, employment, and recidivism. American Sociological Review, 67, 529-546.

Uggen, C., \& Thompson, M. (2003). The socioeconomic determinants of illgotten gains: within-person changes in drug use and illegal earnings. American Journal of Sociology, 109, 146-185.

Viscusi, K. (1986). The risks and rewards of criminal activity : a comprehensive test of criminal deterrence. Journal of Labor Economics, 4, 317-340.

Warr, M. (1996). Organization and instigation in delinquent groups. Criminology, 34, 11-37. 
Warr, M. (1998). Life-course transitions and desistance from crime. Criminology, $36,183-216$.

Warr, M. (2002). Companions in crime: the social aspects of criminal conduct. Cambridge: Cambridge University Press.

Wilson, J.Q., \& Abrahamse, A. (1992). Does crime pay? Justice Quarterly, 9, 359377.

Zaitch, D. (2002). Trafficking cocaine: columbian drug entrepreneurs in the Netherlands. The Hague: Kluwer Law International.

\section{A N NEXE}

Matrice de corrélations entre les variables sélectionnées pour les modèles d'analyse $(n=156)$

\begin{tabular}{|l|c|c|c|c|c|c|c|c|c|}
\hline & 1 & 2 & 3 & 4 & 5 & 6 & 7 & 8 & 9 \\
\hline 1. Revenus criminels ajustés & - & & & & & & & & \\
\hline 2. Âge &, $299^{* * *}$ & - & & & & & & & \\
\hline 3. Revenus légitimes ajustés &,- 060 &, 017 & - & & & & & & \\
\hline $\begin{array}{l}\text { 4. Délits contre les biens } \\
\text { (lambdas ajustés) }\end{array}$ &, $267^{* *}$ &,$- 215^{* *}$ &,- 012 & - & & & & & \\
\hline $\begin{array}{l}\text { 5. Délits de trafic } \\
\text { (lambdas ajustés) }\end{array}$ &, $444^{* * *}$ &,- 141 &, 072 &,- 097 & - & & & & \\
\hline $\begin{array}{l}\text { 6. Proportion de jours de } \\
\text { privation de liberté }\end{array}$ &, 095 &,- 067 &,$- 159^{*}$ &, 103 &,- 027 & - & & & \\
\hline $\begin{array}{l}\text { 7. Durée de la sentence } \\
\text { actuelle }\end{array}$ &, 004 &,- 118 &, 073 &,- 142 &,- 024 &,- 031 & - & & \\
\hline $\begin{array}{l}\text { 8. Capital social des réseaux } \\
\text { de co-délinquance }\end{array}$ &, $308^{* * *}$ &,$- 236^{* *}$ &, $192^{*}$ &, 035 &, $277^{* * *}$ &, 022 &,- 060 & - & \\
\hline $\begin{array}{l}\text { 9. Inaptitude à se } \\
\text { contrôler }\end{array}$ & $438^{* * *}$ & $-309^{* * *}$ &, 008 &, $300^{* * *}$ &, $323^{* * *}$ &, $197^{* *}$ &,- 077 &, $210^{* *}$ & - \\
\hline
\end{tabular}

${ }^{*} p<0,05 ;{ }^{* *} p<0,01 ;{ }^{* * *} p<0,001$ 